\title{
Clinical Neurophysiology: An Overview
}

\author{
Edward C. Mader, Jr., Daniella Miller, and Piotr W. Olejniczak
}

\section{CLINICAL NEUROPHYSIOLOGIC TESTING}

Clinical neurophysiology (CNP) is a time-honored medical specialty that continues to make great strides, bolstered by rapid advances in neuroscience, biomedical engineering, and computer technology. It encompasses a wide range of methods and techniques for recording, presenting, and analyzing neurophysiologic signals in order to diagnose sensory, motor, autonomic, and central nervous system disorders. Testing performed in CNP or procedures used in current neurological practice include a variety of modality-specific and mixed-modality tests (Table 1.1).

Modality-specific CNP tests are performed to assess specific functional modalities using biomedical instruments that measure changes in neurophysiologic signals that occur spontaneously or with activation. Spontaneous fluctuations in electrical and magnetic fields during brain cortical activity are detected with EEG and magnetoencephalography (MEG) $(1,2)$. Changes in electrical potentials with nerve and muscle activity are measured with electromyography (EMG) and nerve conduction studies (NCSs) (3). Subtle changes in the function of signaling pathways can be detected with evoked potentials (EPs), of which the most clinically useful are somatosensory (SSEPs), brainstem auditory (BAEPs), visual (VEPs), and motor (MEPs) evoked potentials (4). SSEPs are elicited by electrical stimulation of the median nerve (M-SSEP), the tibial nerve (T-SSEP), and rarely from other peripheral nerves. MEPs are activated by means of transcranial electrical stimulation (TES-MEP) or transcranial magnetic stimulation (TMS-MEP). The brain's ability to anticipate or respond to cognitive events is examined using event-related potentials (ERPs), such as the Bereitschaftspotential (BSP), contingent negative variation (CNV), P300 (P3), and mismatch negativity (MN) (5). CNP autonomic function Copyright Springer Publishing Company. All Rights Reserved. From: Atlas of Artifacts in Clinical Neurophysiology DOI: $10.1891 / 9780826169358.0001$ testing includes measurement of heart rate variability with deep breathing (HR-DB), recording of blood pressure (BP) and heart rate (HR), response to head-up tilt (HUT) and Valsalva (VAL) maneuver, and sudomotor function by means of the quantitative sudomotor axon reflex test (QSART) and the thermoregulatory sweat test (TST) (6).

Mixed-modality (aka multi-modality) CNP tests utilize two or more test modalities to assess complex states (e.g., sleep, coma), to track multiple physiologic parameters, or to obtain more accurate results. During sleep studies, scalp EEG, chin EMG, and EOG (electrooculogram) allow tracking of sleepwake stages; additional physiologic processes, such as nasal airflow, ventilatory effort, snoring, blood oxygen saturation, ECG, and anterior tibialis EMG, are recorded during polysomnography (PSG) (7). As a rule, neurophysiologic intraoperative monitoring (NIOM) is unimodal with the test modality chosen based on the structures at risk during the procedure, but multimodal NIOM may be superior for selected surgical procedures, such as combining SSEP and MEP monitoring during surgery on the spine (8). In multimodal neuromonitoring (MNM), cerebral blood flow, brain tissue oxygenation, and brain tissue metabolism are monitored, in addition to intracranial pressure (ICP) and EEG, to improve outcome in acute brain injury (9). The multimodal approach, known as coregistration, involves superimposing the data of two or more test modalities to achieve a level of accuracy that is not attainable if each modality is analyzed separately (10). Examples of coregistration techniques are electric source imaging (ESI), magnetic source imaging (MSI), and combined electric-magnetic source imaging (EMSI)

Specific test methods or protocols are selected based on test objectives, patient characteristics, clinical setting, and other factors (11-14). While the routine approach is sufficient in most cases, some clinical problems and 
TABLE 1.1: Clinical Neurophysiologic Tests in Current neurological Practice

\begin{tabular}{|c|c|}
\hline $\begin{array}{l}\text { EEG } \\
\text { Routine scalp EEG (adult/pediatric/neonatal) } \\
\text { Ambulatory scalp EEG monitoring } \\
\text { Electrocorticography } \\
\text { Stereo-electroencephalography } \\
\text { Epilepsy monitoring unit EEG } \\
\text { ICU-EEG monitoring } \\
\text { Intraoperative EEG monitoring }\end{array}$ & $\begin{array}{l}\text { Cortical electrical potential (EEG) } \\
\text { EEG recorded on the scalp } \\
\text { EEG recorded with subdural electrodes } \\
\text { EEG recorded with depth electrodes } \\
\text { EEG recorded on the scalp or intracranially } \\
\text { Same as scalp EEG } \\
\text { Same as scalp EEG }\end{array}$ \\
\hline $\begin{array}{l}\text { MEG } \\
\text { Spontaneous MEG } \\
\text { Evoked MEG }\end{array}$ & $\begin{array}{l}\text { Cortical magnetic flux density (MEG) } \\
\text { Cortical magnetic flux density (MEG) }\end{array}$ \\
\hline $\begin{array}{l}\text { EMG } \\
\text { Needle EMG } \\
\text { Single-fiber EMG } \\
\text { Surface EMG }\end{array}$ & $\begin{array}{l}\text { Motor unit action potential } \\
\text { Single-muscle-fiber action potential } \\
\text { Myogenic electrical potential (surface EMG) }\end{array}$ \\
\hline $\begin{array}{l}\text { NCS } \\
\text { Sensory nerve conduction studies } \\
\text { Motor nerve conduction studies } \\
\text { Including repetitive stimulation and F-wave studies } \\
\text { Sensory-motor reflex studies including H reflex, blink reflex, and } \\
\text { LLR studies } \\
\text { Intraoperative CMAP monitoring }\end{array}$ & $\begin{array}{l}\text { Sensory nerve action potential } \\
\text { CMAP: } M \text { wave } \\
\text { CMAP: } M \text { wave, } F \text { wave } \\
\text { CMAP: } H \text { wave } \\
\text { CMAP: R1 and R2 responses, LLR response } \\
\text { Same as M-NCS }\end{array}$ \\
\hline $\begin{array}{l}\text { EP studies } \\
\text { Pattern-shift VEPs } \\
\text { Flash VEPs FVEPs } \\
\text { BAEPs } \\
\text { SSEP via median/tibial nerve stimulation } \\
\text { MEP via transcranial electric/magnetic stimulation } \\
\text { Intraoperative MEP monitoring } \\
\text { Intraoperative SSEP monitoring } \\
\text { Intraoperative BAEP monitoring } \\
\text { Intraoperative FVEP monitoring }\end{array}$ & $\begin{array}{l}\text { Cortical electrical potential (P100, N100) } \\
\text { Electrical potentials I, II, III, IV, V, VI } \\
\text { Auditory nerve (I), brainstem (II-V) potential } \\
\text { Peripheral nerve (N9/N8), cord (N13/N22), } \\
\text { subcortical (P14, N18/P31, N34), cortical (N20/P37) } \\
\text { Direct wave, indirect waves, and } \\
\text { CMAP: M wave } \\
\text { Same as MEP } \\
\text { Same as M-SSEP/T-SSEP } \\
\text { Same as BAEP } \\
\text { Same as FVEP }\end{array}$ \\
\hline
\end{tabular}


TABLE 1.1: Clinical Neurophysiologic Tests in Current Neurological Practice(continued)

\begin{tabular}{|c|c|}
\hline $\begin{array}{l}\text { Event-related potential studies } \\
\text { Readiness or BSP } \\
\text { CNV } \\
\text { Oddball paradigm } \\
\text { MN }\end{array}$ & $\begin{array}{l}\text { Cortical electrical potential (BSP or premotor potential) } \\
\text { Cortical electrical potential (CNV) } \\
\text { Cortical electrical potential (P300 or P3) } \\
\text { Cortical electrical potential (MN) }\end{array}$ \\
\hline $\begin{array}{l}\text { Autonomic function tests } \\
\text { HR variability with deep breathing } \\
\text { Head-up tilt or tilt table test } \\
\text { Valsalva maneuver } \\
\text { Quantitative sudomotor axon reflex test } \\
\text { Thermoregulatory sweat test }\end{array}$ & $\begin{array}{l}\text { Cardiac electric potential (ECG) } \\
\mathrm{BP}, \mathrm{HR} \\
\mathrm{BP}, \mathrm{HR} \\
\text { Sweat volume } \\
\text { Sweat distribution }\end{array}$ \\
\hline $\begin{array}{l}\text { Polygraphic sleep studies } \\
\text { PSG } \\
\text { Multiple sleep latency test } \\
\text { Maintenance of wakefulness test }\end{array}$ & $\begin{array}{l}\text { Sleep stage: scalp EEG, chin EMG, and EOG } \\
\text { Other parameters (PSG): respiratory airflow and effort, blood } \\
\text { oxygenation, ECG, tibialis anterior EMG, etc. }\end{array}$ \\
\hline $\begin{array}{l}\text { Multimodal neuromonitoring } \\
\text { Multimodal intensive care neuromonitoring } \\
\text { Multimodal intraoperative neuromonitoring }\end{array}$ & $\begin{array}{l}\text { Intracranial pressure, brain perfusion, oxygenation, etc. } \\
\text { Two or more types of signal or test parameters }\end{array}$ \\
\hline $\begin{array}{l}\text { Multimodal coregistration } \\
\text { Electric source imaging } \\
\text { Magnetic source imaging } \\
\text { Electric and magnetic source imaging }\end{array}$ & $\begin{array}{l}\text { EEG coregistered with brain neuroimage } \\
\text { MEG coregistered with brain neuroimage } \\
\text { EEG and MEG coregistered with brain neuroimage }\end{array}$ \\
\hline
\end{tabular}

Note: The first seven categories comprise modality-specific tests, and the last three categories consist of mixed-modality tests (see text for explanation). Listed under each test category are test methods or protocols aimed at specific clinical settings and test objectives. The right column shows the signal of interest or physiologic parameter(s) recorded during neurophysiologic testing.

BAEP, brainstem auditory evoked potential; BP, blood pressure; BSP, Bereitschaftspotential; CMAP, compound motor action potential; CNV, contingent negative variation; EMG, electromyography; EOG, electrooculogram; EP, evoked potential; FVEP, flash visual evoked potential; HR, heart rate; LLR, long-latency reflex; MEG, magnetoencephalography; MEP, motor evoked potential; MN, mismatch negativity; M-NCS, motor nerve conduction study; M-SSEP, median nerve somatosensory evoked potentials; NCS, nerve conduction study; PSG, polysomnography; SSEP, somatosensory evoked potentials; T-SSEP, tibial nerve somatosensory evoked potentials; VEP, visual evoked potentials. 
situations require modifications or refinements in the routine method. For example, the following EEG methods and protocols are aimed at different clinical scenarios: routine scalp EEG, extended EEG recording, ambulatory EEG, neonatal EEG, brain death study, ICU-EEG monitoring, IOM-EEG monitoring, long-term monitoring (LTM) of video and EEG in the epilepsy monitoring unit (EMU), electrocorticography (ECoG), stereo-EEG, and coregistration techniques, such as ESI and EMSI. It may be prudent to combine different test modalities to obtain more accurate results, such as simultaneous EEG and EMG recording to better define the seizure type and EEG recording during HUT to distinguish postsyncopal convulsions from epileptic seizures and psychogenic events.

CNP tests measure specific biosignals or physiological parameters (Table 1.1) allowing clinicians to probe and assess selected facets of the patient's functional anatomy (Table 1.2). The biosignals or physiological parameters recorded reflect the function of specific anatomical structures (Table 1.3). Based on recorded signal characteristics (Figures 1.2 and 1.3), the clinical neurophysiologist can tell if anatomical structures, circuits, or pathways are functioning normally or are affected by pathological processes (Tables 1.4 and 1.5).

Brain function is reflected in the EEG, MEG, ERP, and EP cortical and subcortical components $(1,2,6,8)$. Even when EEG and MEG signals arise from the same cellular processes in the cerebral cortex, there are differences in the way EEG and MEG data are recorded and interpreted (15). A reference is required to record EEG, but not MEG. Because the skull and scalp do not distort magnetic fields as much as electrical fields, the spatial resolution of MEG is superior to EEG $(1,2)$. EEG can detect activity in "more" brain areas by recording radial dipoles, but MEG is more sensitive to tangential dipolar activity in the superficial cortical sulci, making it superior for source localization; for example, MEG is used for MSI and more precise localization of the epileptogenic focus (2). While EEG is widely used in clinical practice, MEG is available only in far fewer centers. The constant interaction between cortical and subcortical structures implies that diencephalic-brainstem disturbances will also affect the EEG/MEG. Assessment of modality-specific brain structures is performed with VEP, BAEP, SSEP, LLR, ERP, and other CNP tests (see later) (3-6). Brain function assessment is the goal of IOM-EEG, sleep studies, and MNM of cerebral perfusion, oxygenation, and metabolism (1,7-9).

Muscle, peripheral nerve, and lower motor neuron function is routinely tested with needle EMG and NCS $(3,16)$. During needle EMG, motor unit action potential (MUAP) number and firing rate increase with the force of muscle contraction. Each MUAP represents activation of a motor unit (i.e., one motor neuron, its nerve fiber, and all muscle fibers innervated by branches of the same nerve fiber). NCS is performed to assess signal conduction along fast-conducting large-diameter nerve fibers: S-NCS for sensory and M-NCS for motor nerve fibers. Neuromuscular junction transmission is evaluated by repetitive nerve stimulation and single-fiber EMG $(3,16)$. F-wave recording extends the utility of M-NCS for evaluating lower motor neurons, motor nerve roots, and proximal motor nerves. These motor structures, along with the sensory nerve roots and central components of reflex pathways, can be evaluated with the H reflex, long-latency reflex, or blink reflex $(3,17)$.

Modality-specific neural pathway or circuit activity is reflected in the EP or ERP signals that are generated when components of the pathway or circuit are activated $(4,5,8)$. The dorsal column pathway is evaluated with M-SSEP and T-SSEP (18), the visual pathway with pattern-shift or flash VEP (19), the auditory pathway with BAEP (20), and the central motor pathway with TES-MEP or TMS-MEP (21). IOM-SSEP and IOM-MEP can be combined if both dorsal and ventral territories of the spinal cord are surgically at risk (22). The neurophysiological substrates of ERP have not yet been identified with certainty. BSP and CNV correlate with movement preparation and motor processing in the frontal lobe, P300 with selective attention and stimulus discrimination in the parietal lobe, and $\mathrm{MN}$ with sound feature discrimination, sensory learning, and perceptual accuracy $(5,23)$.

Autonomic regulation of somatic and visceral function by the parasympathetic and sympathetic nervous system is mediated via slow-conducting small-fiber autonomic nerves that innervate most organs and tissues $(6,24)$. Some autonomic function tests are organ specific, such as pupillometry, lacrimation tests, tests of saliva production, gastric motility tests, and urodynamic studies. In CNP, autonomic testing is performed to evaluate parasympathetic cardiovagal reflexes (HR-DB and VAL), sympathetic vasomotor reflexes (VAL and HUT), and sympathetic sudomotor function (QSART and TST). QSART evaluates the sympathetic axon reflex and TST evaluates the integrity of the sympathetic thermoregulatory pathway from the hypothalamus to eccrine sweat glands $(6,24)$.

The sensitivity of CNP tests is remarkable and most tests will show abnormal results before the onset of clinical signs and symptoms. Unfortunately, CNP tests are also sensitive to technical factors and to noise. Exceptional care should therefore be exercised always to avoid overinterpreting neurophysiologic data. The specificity of CNP tests is limited to the identification of physiological disturbances or disorders in functional anatomy. To reach an etiologic diagnosis, neurophysiological data should always be interpreted in the context of the clinical history, physical examination, and other laboratory test results. 
TABLE 1.2: Classification of Clinical Neurophysiologic Tests Based on Functional Anatomy or Neural Pathway Tested

Tests of global/focal cortical, diencephalic, and/or brainstem function

EEG-spontaneous, scalp-based cortical activity, including influence of diencephalic and brainstem structures

ECoG-focal/regional brain activity, including influence of diencephalic and brainstem structures

Stereo-EEG-focal limbic or deep-brain activity influenced by other cortical and noncortical structures

Epilepsy monitoring unit EEG-same as EEG, ECoG, or stereo-EEG

ICU-EEG-same as EEG

IOM-EEG-same as EEG

MEG-same as EEG, but superior for detecting localized cortical activity

MNM-global cerebral perfusion, oxygenation, metabolism, neocortical activity (see EEG)

Sleep studies-same as EEG, with emphasis on the function of the brain's sleep and wake centers

Tests of muscle, peripheral nerve, motor neuron, and/or reflex function

Needle EMG-motor units activated by volition, spontaneous activity of muscle fibers/motor units

Single-fiber EMG-neuromuscular junction and muscle fiber activity

Surface EMG-multiple motor units activated spontaneously or volitionally

S-NCS-large-fiber sensory peripheral nerve fibers

M-NCS-large-fiber motor peripheral nerve fibers, neuromuscular junction, and muscle fibers

Repetitive stimulation-same as motor NCS, with emphasis on neuromuscular junction transmission

F-wave study-large-fiber motor peripheral nerve fibers, muscle fibers, multiple motor units

IOM-CMAP-same as motor NCS

$\mathrm{H}$ reflex-large-fiber peripheral sensory nerve fibers and multiple motor units ( $\mathrm{H}$ wave)

Long-latency reflex-large-fiber sensory pathway, somatomotor cortex, corticospinal tract, multiple motor units

Blink reflex-trigeminal nerve $\mathrm{V}_{1}$ branch, nuclei and tracts in pons and medulla, facial nerve, orbicularis oculi fibers

Tests of central sensory/motor pathway and/or focal cortical function

VEP-QSART, quantitative sudomotor axon reflex testion, visual cortex

BAEP-auditory pathway: ear, auditory nerve, cochlear nucleus, lateral lemniscus, inferior colliculus

M-SSEP-somatosensory pathway: median nerve, dorsal column, medial lemniscus, thalamus, somatosensory cortex

T-SSEP-somatosensory pathway: tibial nerve, dorsal column, medial lemniscus, thalamus, somatosensory cortex

TES-MEP-motor pathway: motor cortex, corticospinal tract, multiple motor units

TMS-MEP-motor pathway: motor cortex, corticospinal tract, multiple motor units

IOM-SSEP-same as M-SSEP/T-SSEP

IOM-MEP-same as TES-MEP/TMS-MEP

BSP-frontal lobe premotor cortex circuits involved with movement preparation and execution

CNV-frontal lobe premotor cortex circuits involved with movement preparation

P300-parietal lobe circuits for selective attention and stimulus discrimination

$\mathrm{MN}$-temporal lobe auditory cortex circuits for sound feature discrimination

$\mathrm{ESI}, \mathrm{MSI}$, or EMSI-focal area of cerebral cortex generating signal of interest (e.g., epileptiform discharges) 
TABLE 1.2: Classification of Clinical Neurophysiologic Tests Based on Functional Anatomy or Neural Pathway Tested (continued)

\section{Tests of autonomic function}

HR-DB-parasympathetic cardiovagal reflexes

HR-VAL-parasympathetic cardiovagal pathways

BP-VAL-sympathetic baroreflex afferent and efferent pathways

BP-HUT-sympathetic baroreflex afferent and efferent pathways

QSART-sympathetic postganglionic sudomotor axon reflex

TST-sympathetic central, preganglionic, postganglionic pathways and eccrine sweat glands

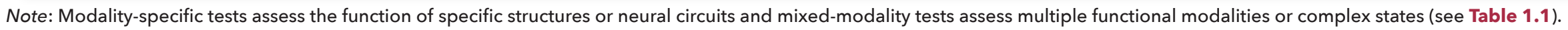

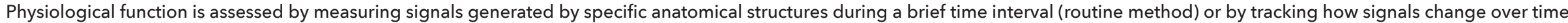
(continuous monitoring).

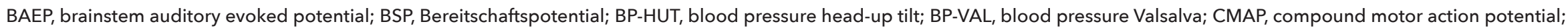
CNV, contingent negative variation; ECoG, electrocorticography; EMG, electromyography; EMSI, Electric and magnetic source imaging; ESI, electric source imaging; HR-DB, heart rate

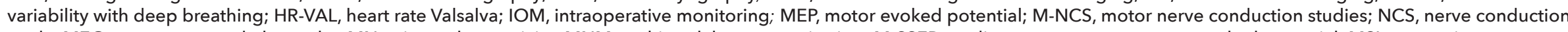
study; MEG, magnetoencephalography; MN, mismatch negativity; MNM, multimodal neuromonitoring; M-SSEP, median nerve somatosensory evoked potential; MSI, magnetic source imaging; QSART, quantitative sudomotor axon reflex test; S-NCS, sensory nerve conduction study; T-SSEP, tibial nerve somatosensory evoked potential; TES-MEP, transcranial electrical stimulation MEP; TMS-MEP, transcranial magnetic stimulation motor evoked potential; TST, thermoregulatory sweat test; VEP, visual evoked potential.

TABLE 1.3: Generators and Sources of Signal and Test Parameters Measured in Clinical Neurophysiology

\begin{tabular}{|c|c|c|}
\hline $\begin{array}{l}\text { EEG/MEG } \\
\text { EEG, ECoG } \\
\text { MEG }\end{array}$ & $\begin{array}{l}\text { Cortical electrical potential }{ }^{a} \\
\text { Cortical magnetic flux density }\end{array}$ & $\begin{array}{l}\text { Cortical pyramidal cell synaptic potential } \\
\text { Cortical pyramidal cell electric currents }\end{array}$ \\
\hline $\begin{array}{l}\text { EMG/NCS } \\
\text { Needle EMG } \\
\text { Single-fiber EMG } \\
\text { Surface EMG }\end{array}$ & $\begin{array}{l}\text { Motor unit action potential } \\
\text { MFAP } \\
\text { Myogenic electric potential }\end{array}$ & $\begin{array}{l}\text { MFAP } \\
\text { MFAP } \\
\text { MFAP }\end{array}$ \\
\hline $\begin{array}{l}\text { Sensory NCS } \\
\text { Motor NCS } \\
\text { F waves } \\
\text { H reflex } \\
\text { Long-latency reflex } \\
\text { Blink reflex }\end{array}$ & $\begin{array}{l}\text { Sensory nerve action potential } \\
\text { CMAPc } \\
\text { CMAP: } F \text { waves }^{b} \\
\text { CMAP: H response } \\
\text { CMAP: LL responses } \\
\text { CMAP: } R 1 \text { and R2 responses }\end{array}$ & $\begin{array}{l}\text { Nerve fiber action potential } \\
\text { MFAP } \\
\text { MFAP } \\
\text { MFAP } \\
\text { MFAP } \\
\text { MFAP }\end{array}$ \\
\hline
\end{tabular}


TABLE 1.3: Generators and Sources of Signal and Test Parameters Measured in Clinical Neurophysiology (continued)

\begin{tabular}{|c|c|c|}
\hline EP/ERP & & \\
\hline VEP & Cortical potential (P100, N100) & Cortical pyramidal cell synaptic potential \\
\hline $\begin{array}{l}\text { FVEP } \\
\text { BAEP }\end{array}$ & $\begin{array}{l}\text { Electrical potentials I, II, III, IV, V, VIc } \\
\text { Auditory nerve action potential (I) } \\
\text { Brainstem farfield potential (IIc, III, IV, V) }\end{array}$ & $\begin{array}{l}\text { Nerve fiber and synaptic potentials } \\
\text { Nerve fiber action potential } \\
\text { Brainstem electric potential }\end{array}$ \\
\hline M-SSEP & $\begin{array}{l}\text { Peripheral nerve potential (N9) } \\
\text { Cervical cord dorsal horn potential (N13) } \\
\text { Subcortical potential (P14, N18) } \\
\text { Somatosensory cortical potential (N20) }\end{array}$ & $\begin{array}{l}\text { Nerve fiber action potential } \\
\text { Dorsal horn cell synaptic potential } \\
\text { Synaptic/action potential } \\
\text { Cortical pyramidal cell synaptic potential }\end{array}$ \\
\hline T-SSEP & $\begin{array}{l}\text { Peripheral nerve potential (N8) } \\
\text { Thoracic cord dorsal horn potential (N22) } \\
\text { Subcortical potential (P31, N34)c } \\
\text { Somatosensory cortical potential (P37) }\end{array}$ & $\begin{array}{l}\text { Nerve fiber action potential } \\
\text { Dorsal horn cell synaptic potential } \\
\text { Synaptic/action potential } \\
\text { Cortical pyramidal cell synaptic potential }\end{array}$ \\
\hline MEP & $\begin{array}{l}\text { Pyramidal tract potential (D wave, I waves) } \\
\text { Compound motor action potential (CMAP) }\end{array}$ & $\begin{array}{l}\text { Nerve fiber action potential } \\
\text { MFAP }\end{array}$ \\
\hline ERP & Cortical potential & Cortical pyramidal cell synaptic potential \\
\hline $\begin{array}{l}\text { Autonomic tests } \\
\text { HR-DB } \\
\text { HUT/VAL } \\
\text { OSART/TST }\end{array}$ & $\begin{array}{l}\text { Electrocardiographic potential (ECG) } \\
\text { Arterial BP (mmHg), heart rate (/min) } \\
\text { Eccrine gland output (sweat) }\end{array}$ & $\begin{array}{l}\text { Cardiac MFAP } \\
\text { Not applicable } \\
\text { Not applicable }\end{array}$ \\
\hline
\end{tabular}


TABLE 1.3: Generators and Sources of Signal and Test Parameters Measured in Clinical Neurophysiology (continued)

\begin{tabular}{|c|c|c|}
\hline Sleep studies & & \\
\hline $\begin{array}{l}\text { EEG (scalp) } \\
\text { EMG (chin) } \\
\text { EOG (near eye) }\end{array}$ & $\begin{array}{l}\text { Cortical electric potentiala } \\
\text { Myogenic electric potential } \\
\text { Oculoelectric dipole }\end{array}$ & $\begin{array}{l}\text { Cortical pyramidal cell synaptic potential } \\
\text { MFAP } \\
\text { Corneal-retinal potential }\end{array}$ \\
\hline Other parameters ${ }^{d}$ & $\begin{array}{l}\text { Respiratory airflow } \\
\text { Respiratory effort } \\
\text { Blood oxygenation } \\
\text { Cardioelectric potential (ECG) } \\
\text { Tibialis anterior muscle potential }\end{array}$ & $\begin{array}{l}\text { Not applicable } \\
\text { Not applicable } \\
\text { Not applicable } \\
\text { Cardiac MFAP } \\
\text { MFAP }\end{array}$ \\
\hline $\begin{array}{l}\text { Neuromonitoring } \\
\text { Unimodal NIOM } \\
\text { Multimodal NIOM MNM }\end{array}$ & $\begin{array}{l}\text { Same as EEG, CMAP, MEP, SSEP, etc. } \\
\geq 2 \text { modalities, e.g., IOM-SSEP and MEP } \\
\text { ICP, brain perfusion, brain tissue oxygenation, cellular } \\
\text { metabolism, etc. }\end{array}$ & $\begin{array}{l}\text { Same as modality-specific test } \\
\text { Same as modality-specific test } \\
\text { Not applicable } \\
\text { Not applicable }\end{array}$ \\
\hline $\begin{array}{l}\text { Coregistration } \\
\text { ESI } \\
\text { MSI } \\
\text { EMSI }\end{array}$ & $\begin{array}{l}\text { Same as EEG + electromagnetic } \\
\text { Same as MEG + electromagnetic } \\
\text { Same as EEG and MEG + electromagnetic }\end{array}$ & $\begin{array}{l}\text { See EEG above } \\
\text { See MEG above } \\
\text { See EEG and MEG above }\end{array}$ \\
\hline
\end{tabular}

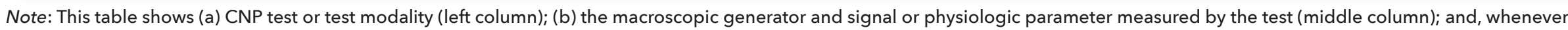
applicable, (c) the microscopic SGU and the microscopic signal or "building block" of the macroscopic signal (right column).

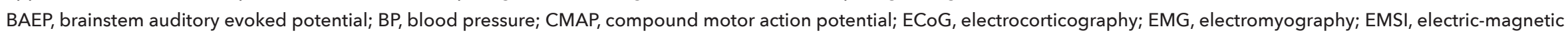

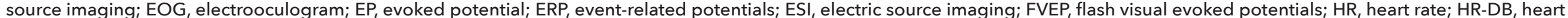

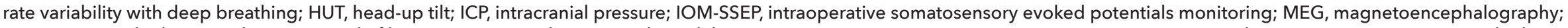
MEP, motor evoked potential; MFAP, muscle fiber action potential; MNM, multimodal neuromonitoring; MSI, magnetic source imaging; M-SSEP, median nerve somatosensory evoked potentials; NCS, nerve conduction study; NIOM, neurophysiologic intraoperative monitoring; QSART, quantitative sudomotor axon reflex test; SGU, signal-generating unit; SSEP, somatosensory evoked potentials; TST, thermoregulatory sweat test; T-SSEP, tibial nerve somatosensory evoked potentials; VAL, Valsalva; VEP, visual evoked potentials.

aNonsynaptic and nonneuronal mechanisms may also play a role in EEG/MEG generation.

bLate responses are CMAPs that occur after the classic CMAP known as M wave.

'Generator(s) of FVEP waves I-VI, BAEP wave II, and SSEP subcortical components remain controversial.

dPhysiologic parameters recorded during PSG in addition to EEG, EOG, and chin EMG (list is incomplete).

Translating bioelectric and biomagnetic signals into physiological information requires a priori knowledge of where and how signals are generated (discussed earlier) and how signals spread from the generator to the recording site. Once generated, bioelectric and biomagnetic fields propagate from the generator to other parts of the body. This mechanism of signal propagation, known as volume conduction, must be distinguished from another mechanism of signal propagation, known as neural transmission. These two mechanisms of signal propagation in biological tissue are quite distinct and result in waveforms with different spatiotemporal characteristics (Figure 1.4). 
TABLE 1.4: Spatiotemporal Characteristics of Bioelectric Signals Measured in Clinical Neurophysiology

\begin{tabular}{|c|c|c|}
\hline EEG & Cortical electric potential ${ }^{a}$ & Stationary nearfield \\
\hline MEG & Cortical magnetic flux density ${ }^{a}$ & Stationary nearfield \\
\hline $\begin{array}{l}\text { Needle EMG } \\
\text { Single-fiber EMG }\end{array}$ & $\begin{array}{l}\text { Motor unit action potential } \\
\text { MFAPb }\end{array}$ & $\begin{array}{l}\text { Traveling nearfield } \\
\text { Traveling nearfield }\end{array}$ \\
\hline $\begin{array}{l}\text { Sensory NCS } \\
\text { Motor NCS } \\
\text { Reflexes: H, LL, and blink }\end{array}$ & $\begin{array}{l}\text { Sensory nerve action potential } \\
\text { CMAPc }^{c} \\
\text { CMAP }^{c}\end{array}$ & $\begin{array}{l}\text { Traveling nearfield } \\
\text { Traveling nearfield } \\
\text { Traveling nearfield }\end{array}$ \\
\hline Pattern-shift VEP & Cortical potential (P100, N100) & Stationary nearfield \\
\hline BAEP & $\begin{array}{l}\text { Auditory nerve action potential (I) } \\
\text { Brainstem farfield potential (IIc, III, IV, V) }\end{array}$ & $\begin{array}{l}\text { Traveling nearfield } \\
\text { Stationary farfield }\end{array}$ \\
\hline M-SSEP/T-SSEP & $\begin{array}{l}\text { Peripheral nerve action potential (N9/N8) } \\
\text { Cervical cord dorsal horn potential (N13/N22) } \\
\text { Subcortical potential (P14, N18/P31, N34)d } \\
\text { Somatosensory cortical potential (N20/P37) }\end{array}$ & $\begin{array}{l}\text { Traveling nearfield } \\
\text { Stationary nearfield } \\
\text { Stationary farfield } \\
\text { Stationary nearfield }\end{array}$ \\
\hline TES-MEP/TMS-MEP & $\begin{array}{l}\text { Corticospinal tract action potential ( } D \text { wave, I waves) } \\
\text { CMAP }\end{array}$ & $\begin{array}{l}\text { Traveling nearfield } \\
\text { Traveling nearfield }\end{array}$ \\
\hline ERP & Cortical electric potential & Stationary nearfield \\
\hline$E C G e$ & Cardiac electric potential & Stationary farfield ${ }^{d}$ \\
\hline
\end{tabular}

aNonsynaptic and non-neuronal mechanisms may play a role in the generation of EEG, MEG, ERPs, and cortical EPs

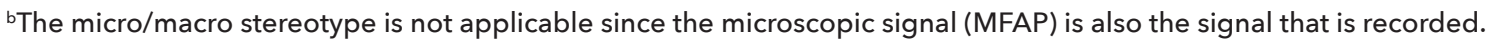

cLate responses ( $F$ waves, $\mathrm{H}$ reflex, LL response, R1/R2 responses) are CMAPs that occur after the classic CMAP or M wave.

dNo definite consensus on the generator(s) of BAEP wave II and SSEP subcortical components and of FVEP waves I-VI

eECG is a traveling nearfield signal near its generator but behaves as a farfield stationary signal when recorded in CNP.

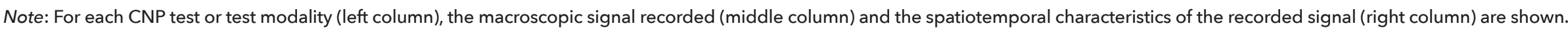
BAEP, brainstem auditory evoked potential; CMAP, compound motor action potential; EMG, electromyography; ERP, event-related potentials; MEG, magnetoencephalography; MFAP, muscle fiber action potential; M-SSEP, median nerve somatosensory evoked potentials; NCS, nerve conduction study; TES-MEP, transcranial electrical stimulation motor evoked potential; TMS-MEP, transcranial magnetic stimulation motor evoked potential; T-SSEP, tibial nerve somatosensory evoked potentials; VEP, visual evoked potentials. 
TABLE 1.5: Classification of Electrophysiologic Abnormalities ${ }^{a}$

\section{Signal amplitude}

\section{Abnormally low amplitude}

Loss of SGUs-e.g., low SNAP with loss of nerve fibers, EEG attenuation with loss of pyramidal cells

Decrease in number of SGUs activated-e.g., low CMAP amplitude with loss of nerve fibers

SGU asynchrony-e.g., low CMAP amplitude due to temporal dispersion of signals in motor nerve fibers

Changes in volume conductor-e.g., edema increasing distance between generator and electrode

Abnormally high amplitude

Increase in number of SGUs activated-e.g., giant MUAP with muscle reinnervation of fibers

Pathologic hypersynchrony of SGUs-e.g., high-amplitude epileptiform waves, giant N20 or P100

Decrease in impedance of conducting medium-e.g., accentuation of fast EEG waveforms with skull defects

\section{Signal conduction time}

\section{Abnormally long latency}

Conduction slowing in peripheral nerve-e.g., prolonged SNAP, CMAP, SSEP, or P100 absolute latency

Conduction slowing in central tract-e.g., increase in BAEP III-V and SSEP N13-N20 interpeak latencies, temporal dispersion and asynchronous activation of SGUs-usually only mild prolongation in latency

Abnormally short latency

Not easy to account for with pathology; a technical reason must be excluded-e.g., electrode placement or artifact

\section{Wave morphology or configuration}

\section{Abnormal wave morphology}

Combination of abnormalities-e.g., wave amplitude, duration, contour, number of phases/turns

Increase in wave duration and number of phases

SGU desynchrony-e.g., long CMAP duration due to temporal dispersion of signals in motor nerve fibers

Short wave duration and sharp contour

SGU hypersynchrony-e.g., EEG and MEG epileptiform discharges, spikes, and sharp waves 
TABLE 1.5: Classification of Electrophysiologic Aabnormalities ${ }^{a}$ (continued)

\section{Wave activation, modulation, and repetition}

\section{Abnormal activation or recruitment}

Reduced SGU activation-e.g., decreased MUAP recruitment with motor neuron or nerve fiber pathology

Increased SGU activation-e.g., increased MUAP recruitment due to muscle fiber pathology

Reduced and increased SGU activation-e.g., release of latent slow rhythms with loss of pyramidal cell input

Abnormal spontaneous activity

Hyperexcitability of SGUs-e.g., fibrillation potentials, positive sharp waves, complex repetitive discharge

Hyperexcitability of non-SGU circuit components-e.g., fasciculation potentials, myokymia

Hyperexcitability of circuit-e.g., EEG or MEG epileptiform activity

Abnormal repetition rate

Perturbation in network dynamics-e.g., disorganization of EEG or MEG rhythms with encephalopathy

\section{Spatial characteristics}

\section{Abnormal spatial distribution}

Often due to the same factors affecting the time-domain characteristics of waves

Apparent displacement of signal generator

Technical factors-e.g., improper electrode placement

State change-e.g., drowsiness can manifest as "anterior displacement" of EEG alpha rhythm.

\section{Clinical and physiological context}

\section{Physiological state}

Often due to the same factors affecting the time-domain characteristics of waves

Age or developmental period

Maturational-e.g., dysmature features in the neonatal EEG

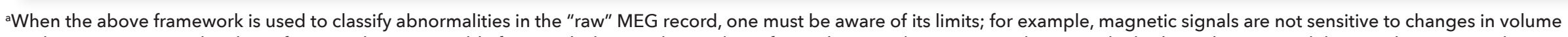
conductor properties. The above framework is not suitable for signals that are the product of complex transduction events between the biological source and the signal-sensing probe.

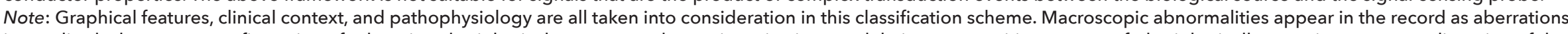

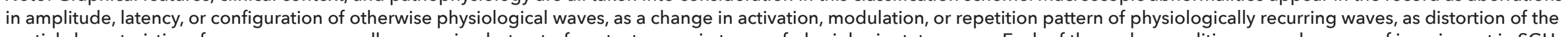

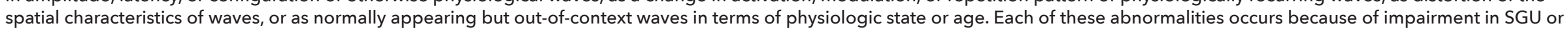
non-SGU circuit components or because of changes in the properties of the volume conductor.

BAEP, brainstem auditory evoked potential; CMAP, compound motor action potential; MEG, magnetoencephalography; MUAP, motor unit action potential; SGU, signal-generating unit; SNAP, sensory nerve action potential; SSEP, somatosensory evoked potentials. 


\section{SIGNAL RECORDING AND GRAPHICAL DISPLAY}

The signal that must be recorded and analyzed, or the "signal of interest," is often simply called "signal." Any other signal contaminating and potentially distorting the signal of interest is considered "noise." Noise entering the recording system may appear on the record as artifact. Because signal and noise are physically similar (both are electromagnetic signals), neurophysiologic instruments are intricately engineered to maximize the signal-to-noise ratio during signal recording and processing (25). Moreover, CNP technologists and specialists are trained to distinguish signal from artifact by visual inspection and, if necessary, digital reformation of the record. Chapter 2 is dedicated to instrumentation and signal processing. In this chapter, it is sufficient to mention that neurophysiological recording involves four processes: analog signal acquisition, analog signal conditioning, analog-to-digital conversion, and digital signal processing (Figure 1.1).

Signal analysis and interpretation is performed by professionals trained in CNP. Neurophysiologic data are usually presented and analyzed in the time domain (Figure 1.2). Recorded signals are displayed as waveforms on a computer or oscilloscopic screen (paper recording is nearly obsolete and is omitted from our discussion). CNP records contain one or more lines of tracing, called channels. Each channel is a graph of signal amplitude versus time, with waves rising above and falling below the baseline, indicating fluctuations in signal amplitude with time. When differential amplification is employed in biopotential recording, the wave amplitude at any given time is proportional to the voltage difference between the amplifier inputs, G1 and G2, multiplied by the amplification factor or gain (see Chapter 2) (25).
Quantitative analysis of "raw" data can extract additional information that can complement or supplement basic time-domain data. The fast Fourier transform (FFT) converts data in the time domain to an alternative representation of the same data in the frequency domain (26). Decomposing EEG into its frequency components and using compressed spectral array (CSA) to track the changes in amplitude/power of EEG frequency bands is a useful quantitative $E E G$ (qEEG) technique for detecting cerebral ischemia and seizures during ICU-EEG monitoring (11). EEG trending in neonates is commonly performed using amplitude-integrated EEG (aEEG), a qEEG technique that involves filtering, rectification, smoothing, and compression of time-domain data. Quantitative analysis of MUAP, compound motor action potential (CMAP), $\mathrm{HR}-\mathrm{DB}$, and other data can also be performed. Although waveform analysis in the time domain is still the bread and butter of the clinical neurophysiologist, rapidly advancing computer technology and machine learning, and the increasing availability of automated algorithms, will inevitably transform the future practice of CNP.

Spontaneously generated signals represent oscillating physiologic processes, appear graphically as sinusoidal waves, and are referred to as rhythmic activity or rhythms (Figure 1.2A) (27). In EEG/MEG, sustained rhythmic activity is considered "background." Background activity, not individual waves, serves as the framework for time-domain analysis. Additional waves and rhythms may appear spontaneously or with activation and are mixed with, or replace, the background. Both background activity and emergent waves are signals of interest that are analyzed collectively. The most important graphical descriptor of rhythmic activity is frequency, the number of wave repeats per second, which is reported as Hertz $(\mathrm{Hz})$ or cycles per second (cps or c/s). Frequency is described in precise terms (e.g., $60 \mathrm{~Hz}$ ) or in terms of frequency bands, including EEG/MEG: less than $4 \mathrm{~Hz}$ is delta, 4 to less than $8 \mathrm{~Hz}$ is theta, 8 to $13 \mathrm{~Hz}$

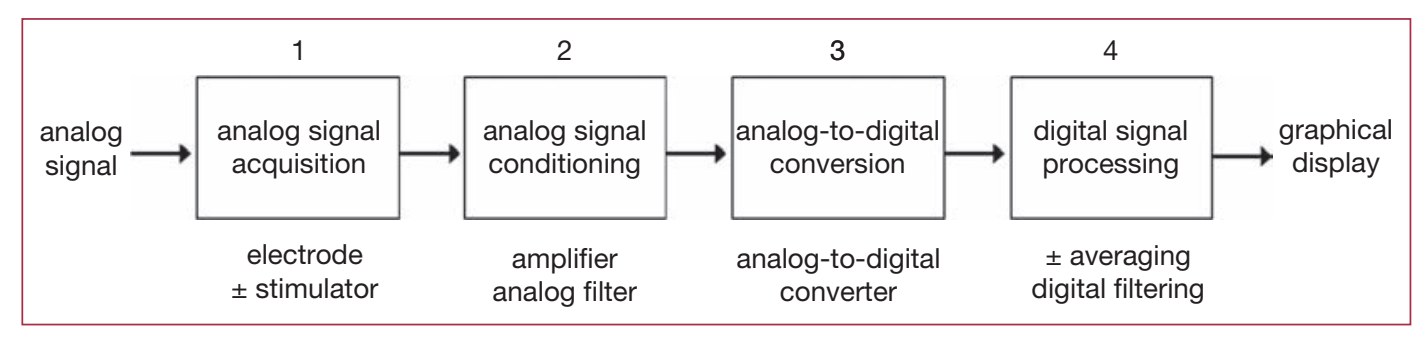

FIGURE 1.1: Signal recording in clinical neurophysiology. The analog signal recorded includes the signal of interest and noise. Signal recording involves four general processes: (1) analog signal acquisition, (2) analog signal conditioning, (3) analog-todigital conversion, and (4) digital signal processing. The end product of the recording process is a graphical representation or record of the signals (see Figure 1.2). 

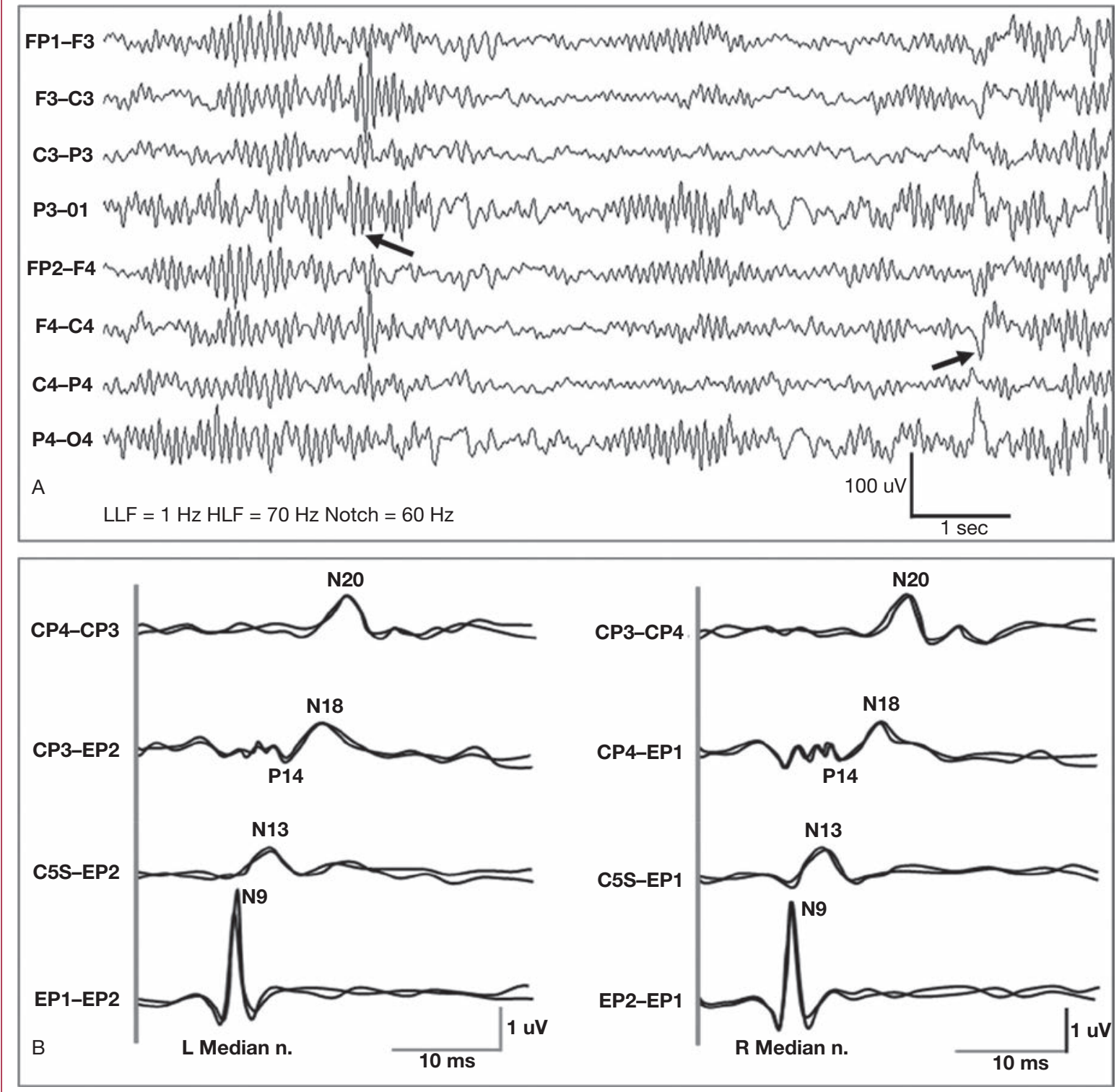

FIGURE 1.2: Graphical display of neurophysiologic signals. Recorded signals are usually displayed as a graph of signal amplitude ( $y$-axis) versus time ( $x$-axis) with each line of tracing (channel) showing how amplitude varies with time. A: Spontaneously generated signals (e.g., scalp EEG) are displayed as continuous tracings. The EEG sample shown is an 8-channel, 10-second epoch extracted from a 30-minute recording using the 10-20 system of electrode placement [11]. Background rhythm (left arrow) and emergent waves (right arrow) on the left (upper four channels) and right (lower four channels) sides of the head are analyzed collectively. The most important descriptor of rhythmic activity is frequency, the number of wave repeats per second, reported as Hertz (Hz). B: Stimulus-evoked signals (e.g., M-SSEP) are displayed as short-interval tracings, called sweeps. In the figure, each line of tracing is the average of 200 sweeps obtained by stimulating the median nerve, one side at a time, and recording from standard sites [11]. Two tracings are overlapped to prove replicability. Five SSEP peaks, N9, N13, $\mathrm{P} 14, \mathrm{~N} 18$, and N20, are identified based on morphology and time of occurrence. The absolute and interpeak latencies and the degree of left-right asymmetry in SSEP latencies are analyzed using laboratory-specific normative values.

M-SSEP, median nerve somatosensory evoked potential. 
is alpha, and greater than $13 \mathrm{~Hz}$ is beta (28). By interpreting a wave as a basic unit of a rhythm, an isolated waveform can be assigned a frequency that is equal to the reciprocal of its duration.

Stimulus-evoked signals, representing neurophysiological events that occur in response to specific stimuli, are typically displayed as waveform tracings one sweep at a time (Figure 1.2B) (27). The evoked waves or wave complexes are stereotypical-they are activated only by appropriate stimulus, they are time-locked to the stimulus, and they have a distinctive graphical appearance (29). While a single acquisition sweep is unlikely to capture very low amplitude signals that are buried in noise, averaging many sweeps can increase the signal-to-noise ratio and reveal these tiny signals. Latency is the time between two events. It is called absolute latency if the first event is a stimulus presentation and the second event is wave onset (onset latency) or wave peak or trough (peak latency), or interpeak latency if both events are wave peaks or troughs. Duration is the time from the onset to the termination of a wave or wave complex. Phase is the portion of the wave between two baseline crossings. Turns or serrations are reversals in the direction a waveform "flows" relative to the baseline. The amplitude, duration, and number of phases and turns of a wavelet or waveform complex determine its configuration or morphology.

CNP test result interpretation is the artful integration of clinical, technical, and graphical information. Most CNP records are simple twodimensional graphs of signal amplitude versus time (Figure 1.2). The optimal display configuration, including number of channels, montage, sensitivity, time base, and filter settings, is different for each CNP test modality or procedure (Figure 1.3) (1-15). Interpreting CNP records requires skills and abilities in pattern recognition, spatial localization, contextual analysis, visual approximation, and computer-assisted measurements. The following is an overview of the common graphical display and analysis methods employed in CNP practice.

$E E G / M E G$ signals are simultaneously recorded from multiple cranial sites and displayed as a multichannel record (Figure 1.3: top). Each line of tracing in the EEG record represents the difference in voltage between a pair of electrodes (30). Since MEG is reference free, each line of tracing in the MEG record corresponds to the fluctuation of magnetic flux density detected by a superconducting quantum interference device (SQUID) detector. The "raw" time-domain EEG/MEG record is displayed and read one page (epoch) at a time. In the process, EEG/MEG waves are characterized based on spatial distribution and time-domain characteristics, including amplitude, frequency, polarity, and morphology. Spontaneous and sustained rhythmic activity is considered background activity. Waveforms appearing spontaneously or with activation are analyzed in the context of the background and other waveforms. MEG signals are often processed, coregistered with a neuroimage (e.g., MRI), and displayed as an MSI image (not shown in Figure 1.3) (31).
EMG/NCS signals are acquired with needle or surface electrodes and displayed as a tracing with the option to superimpose or stack several tracings on the screen (Figure 1.3: second row). In needle EMG, a fully relaxed muscle is "silent" if the electrode is not picking up endplate noise (Figure 1.3: EMG relaxed). With slight muscle contraction, an MUAP appears with a low firing rate and, as effort increases, firing rate increases and more MUAPs are recruited (Figure 1.3: EMG activated). Abnormal spontaneous and exertional activity is identified by visualizing the waveforms and listening to the sounds emitted by a synthesizer (32). In M-NCS / S-NCS, electrical stimulation of a motor/sensory peripheral nerve triggers a CMAP/sensory nerve action potential (SNAP), which is displayed on the screen one sweep at a time (Figure 1.3: CMAP, SNAP). Latency and amplitude are measured and conduction velocity is calculated (16).

$E P / E R P$ responses are obtained using standardized activation-recording protocols and displayed as waves that are time-locked to the stimulus (Figure 1.3: third row). Peripheral nerve, spinal cord, subcortical, and cortical SSEPS are evoked with median/tibial nerve stimulation (Figure 1.3: SSEP), cortical VEPS with monocular pattern-shift stimulation (Figure 1.3: VEP), BAEPS with monoaural stimulation (Figure 1.3: BAEP), pyramidal tract and myogenic $M E P S$ with transcranial electric/magnetic stimulation (Figure 1.3: MEP), and cortical ERPS with cognitive processes or tasks (not shown in Figure 1.3). EP/ERP requires signal averaging and juxtaposition of two or more averages to demonstrate replicability. Although the most reliable indicator of pathology in routine EP studies is latency prolongation, the most sensitive indicator of impending injury during EP monitoring is decreasing amplitude of response (33).

Autonomic function test results are commonly displayed as graphs in the time domain (Figure 1.3: bottom) (34). In $H R-D B$, a graph of ECG R-R interval shows how HR changes with respiration (Figure 1.3). The BP and HR are plotted as a function of expiratory pressure in VAL (Figure 1.3) and as a function of the time of tilt in HUT (Figure 1.3). The QSART record is a graph of sweat output or rate of sweating in response to iontophoresis of acetylcholine through the skin (Figure 1.3). In TST, a body surface map of sweat density is constructed based on the change in color of an indicator as it reacts with sweat (not shown).

Mixed-modality tests display the results of different test modalities in one record or in separate records (not shown in Figure 1.3). In PSG, cross-modal correlation is facilitated by displaying EEG, chin EMG, EOG, respiratory airflow and effort, oxygen saturation, snoring, ECG, and tibialis anterior EMG in the same record. MNM employs a variety of techniques for monitoring ICP, cerebral hemodynamics, brain tissue oxygenation, brain biochemistry and metabolic markers, EEG, and other parameters. With the advent of multiparameter probes and other advances in biomedical engineering, we can look forward to more integrated and less invasive approaches to MNM (35). 


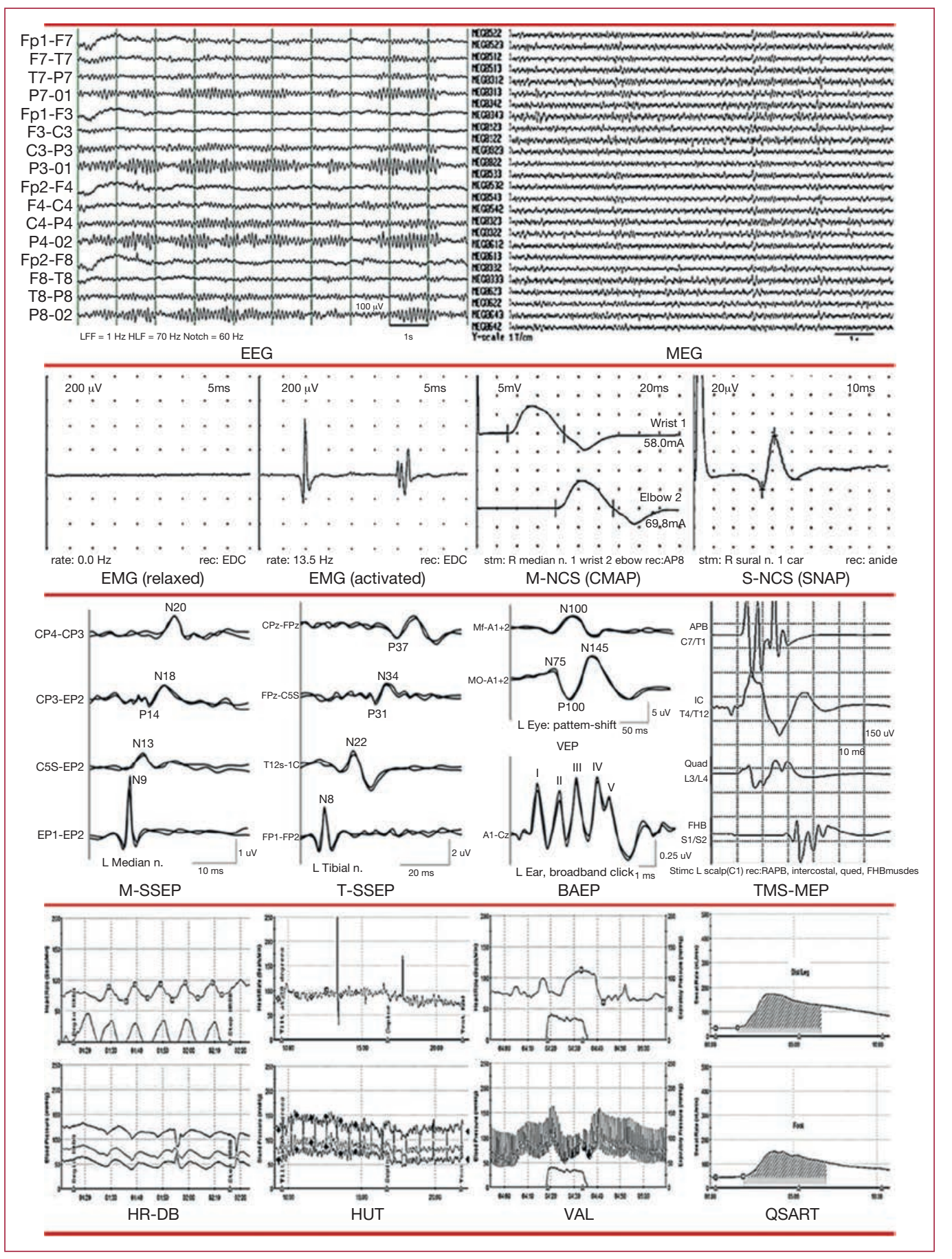

FIGURE 1.3: Clinical neurophysiologic records. Sample records showing how test results are typically displayed in EEG/MEG (top), EMG/NCS (second row), EP studies (third row), and autonomic function tests (bottom). All records are time-domain graphs of signal amplitude ( $y$-axis) versus time (x-axis). Also shown are the amplitude/time scale and, when necessary, the parameter measured, recording site, and channel derivation. Although both sides are tested in routine EMG, NCS, and EP studies, only one side is shown. Image credits: MEG-Dr. Michael M. Quach*; EMG/NCS-Dr. John D. England ${ }^{\star \star}$ and Dr. Christopher O. Edwards s*; MEP-Dr.

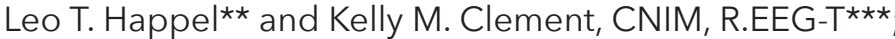
Autonomic testing: HR-DB, HUT, VAL, and QSART-Dr. Paul A. Lelorier**; the rest are from the teaching files of the authors ${ }^{\star \star}$. Institutions: *Baylor College of Medicine, **Louisiana State University Health Sciences Center, ${ }^{\star * *}$ Children's Hospital of New Orleans.

BAEP, brainstem auditory evoked potentials; EMG, electromyography; $E P$, evoked potential; $H R-D B$, heart rate variability with deep breathing; HUT, head-up tilt; MEG, magnetoencephalography; NCS, nerve conduction study; M-NCS, motor nerve conduction study; M-SSEP, median nerve somatosensory evoked potential; OSART, quantitative sudomotor axon reflex test; S-NCS, sensory nerve conduction study; TMS-MEP, transcranial magnetic stimulation motor evoked potential; T-SSEP, tibial nerve somatosensory evoked potential; VAL, Valsalva. 


\section{NORMAL SIGNAL GENERATION AND PROPAGATION}

In CNP, the word generator without a qualifier means signal generator, not rhythm generator. The signal generator is the biological structure directly generating the signal that is recorded by the instrument (36). It can be a gross structure, a circuit or network, a tissue or cell type, or a cell substructure (Table 1.3). The generator paradigm works well if the recorded macroscopic signal represents the linear superposition of many elementary microscopic signals. This is the case with bioelectric and biomagnetic signals in which the macroscopic signal is the sum of many microscopic signals, each of which is contributed by a signal-generating unit (SGU). The notion of a generator is not suitable if the recorded signal is a product of complex transduction events occurring between the biological source and the signal-sensing probe.

Bioelectric and biomagnetic signals can be traced to a change in the membrane potential of cells as ion currents flow across the cell membrane in response to a change in the conductance of ion channels (37). A change in membrane potential (depolarization or hyperpolarization) occurs at a focal site of the membrane (e.g., postsynaptic site) and spreads passively and locally (electrotonic conduction). Spatiotemporal summation of local synaptic potentials can lead to suprathreshold depolarization and trigger an actively propagating potential, known as action potential.

The SGU and elementary signal is firmly established for some CNP signals (Table 1.3). In needle EMG, the MUAP is a compound muscle action potential, the sum of the action potentials of muscle fibers belonging to the same motor unit. The muscle fiber is the SGU and the muscle fiber action potential (MFAP) is the elementary signal producing the MUAP. The signals recorded in NCS are also compound action potentials. Like the MUAP, the CMAP, the $F$ waves, and the $H$ response are the sum of MFAPs. In contrast, the SNAP is the sum of the action potentials of fast-conducting sensory nerve fibers. The nerve fiber is the SGU and the nerve fiber action potential (NFAP) is the elementary signal producing the SNAP. Like the SNAP, the BAEP wave I, the M-SSEP N9, and the T-SSEP N8 are compound nerve action potentials. The direct waves ( $D$ waves) and indirect waves (I waves) in MEP are compound nerve action potentials arising from corticospinal or pyramidal tract fibers. The CMAP in MEP is analogous to the CMAP in NCS. The M-SSEP N13 and the T-SSEP N22 are the sum of dorsal horn cell synaptic potentials and are not generated from the dorsal column (33).

Even if the principal SGU and elementary signal is known, the contribution of other SGUs in shaping the macroscopic signal may not be fully understood (Table 1.3). For example, the main SGU of the EEG is the cortical pyramidal cell and the elementary signal is the postsynaptic potential. There is, however, evidence that glial cells and nonsynaptic neural events, such as action potentials, calcium spikes, voltage-dependent intrinsic oscillations, intrinsic spikes after hyperpolarization, and ephaptic effects, have some role in shaping the EEG (38). The same argument applies to other signals generated in the cerebral cortex, including MEG, ERPs, the VEP P100, the M-SSEP N20, and the T-SSEP P37. There is consensus on the brainstem generators of BAEP waves III, IV, and $V$, but the generator and SGU remain controversial for BAEP wave II and for the subcortical SSEPs P14, N18, N34, and P31. Despite decades of experience with $B S P, C N V, P 300$, and $M N$ recording, the neural generators of cognitive ERPs are still not fully understood.

The generator concept is not suitable for some CNP signals and physiological parameters, such as the HR, BP, and sweat output measured during autonomic testing; the nasal airflow, ventilation, and oxygen saturation detected with PSG; and the ICP, ultrasonic flow and near-infrared signals, and chemical compounds monitored during MNM. The mechanisms by which biophysical parameters (e.g., temperature, pressure, flow, volume, stretch) are transduced to electronic current, processed in the circuitry of the apparatus, and transformed into a readable graph are outside the scope of this chapter (39).

Volume conduction is the propagation of an electrical or magnetic field inside a volume conductor. It can be represented by a simple model, such as a point source generating a field that spreads in a homogeneous infinite volume conductor. More realistic models take into account the complexities of the source (e.g., charge distribution) and the volume conductor (e.g., varying tissue resistance) (40). Biological tissue responds to a propagating field by inducing an electrical current, the magnitude of which is inversely related to tissue resistance. Because the speed of volume conduction is only slightly less than the speed of light in a vacuum, as soon as a field is generated, it will propagate to the rest of the body almost instantaneously. In the case of an electrical field, the potential recorded depends on the distance of the recording site from the generator and on the properties of the generator (e.g., field strength, charge distribution) and the volume conductor (e.g., tissue resistance). The spatial rate of change of electrical potential reflects the shifting properties of tissues that the field encounters as it spreads inside the body. A nearfield signal is characterized by rapid potential drop and substantial loss in signal amplitude even when it is recorded at locations that are not too far from the generator. By contrast, a farfield signal is characterized by a slow potential drop and relative preservation in amplitude when recorded far from the generator (41). The extent of volume conduction (nearfield vs. farfield) and the electrode positions (E1 and E2) determine how much of the electrical potential is recorded (Figure 1.4).

Neural transmission is a physiological process involving excitation of the cell membrane of nerve, muscle, or other excitable cells. Unlike volume 


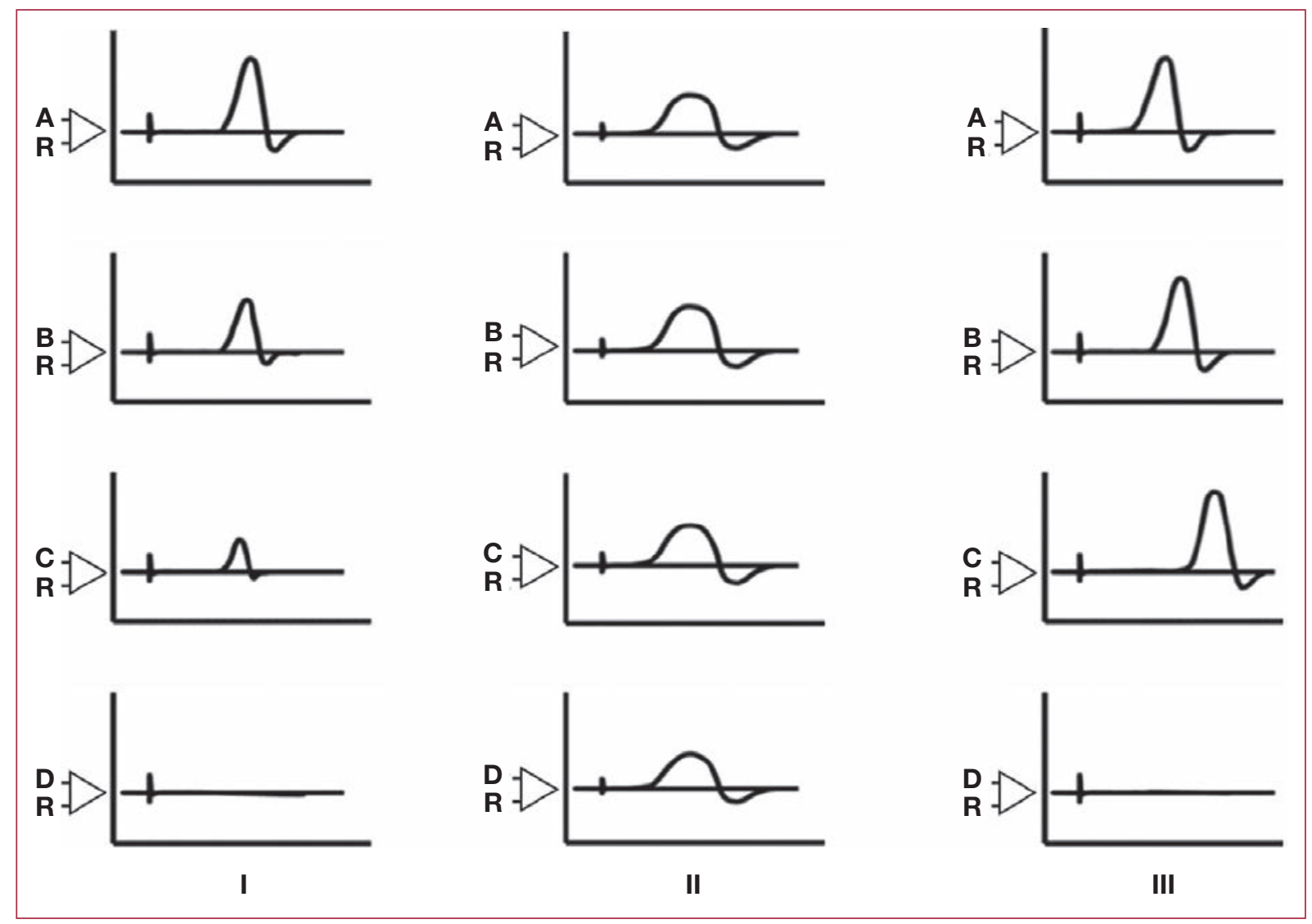

FIGURE 1.4: Spatiotemporal characteristics of signals. Recording the signal close to its generator may be enough to discern its time-domain characteristics but, to appreciate its spatial characteristics, the signal must be recorded from multiple sites ( $A, B, C, D$ in the diagram; $R=$ reference) and the tracings displayed to show the location of signal recording relative to the location of signal generation (top to bottom in diagram). A stationary nearfield signal (I) will exhibit decreasing peak amplitude with increasing distance from the generator $(A-C)$ and disappear when no longer detectable (D). A stationary farfield signal (II) will have no change or a slight change in peak amplitude with increasing distance from the generator (A-D). A signal transmitted via neural mechanisms (III), namely a propagated potential or traveling wave, will show increasing peak latency with increasing distance from the signal generator $(A-C)$ and will not be detected at a site that is located far from the propagating signal (D). 
conduction, neural transmission is the result of active signal generation and regeneration along the cell membrane. Because neural transmission involves active processes, its speed is many orders of magnitude lower than the speed of volume conduction (42). A signal spreading by neural transmission is called a propagating potential or a traveling wave, terms that are both misleading. The electrical potential (potential energy per unit charge) is measured at a point in the field, and its value is always relative to another point (a point at infinity is often used as reference). Although the value of the potential changes from one point to another, it is the electrical field that physically propagates in space. The trace or waveform displayed on each channel represents fluctuations of electrophysiologic potential in time, not in space. Unlike in physics, a traveling wave in CNP is not a wave propagating in a medium but a series of waves that are actively generated each time a different part of the cell membrane is activated (Figure 1.4C).

The spatiotemporal characteristics of a signal can be appreciated if the signal is recorded from a few strategic locations and the tracings are displayed "topographically" (Figure 1.4). The location of the generator is approximated by the site of maximum signal amplitude. A stationary nearfield signal has a peak amplitude that decreases with increasing distance from the generator (Figure 1.4A). The abrupt amplitude drop-off with distance of a nearfield signal may render the signal undetectable a few centimeters from the generator (site D). On the other hand, a stationary farfield signal will show no change or only very slight change in amplitude with increasing distance from the generator (Figure 1.4B). During neural transmission, a "traveling" nearfield signal (usually called "propagating potential" or "traveling wave") will show increasing peak latency as the signal "travels" from the initial generation site onward to a series of recording sites (Figure 1.4C). The signal is undetectable when recorded far from the path of signal transmission (site D). Clinical neurophysiologic testing methods and protocols differ in terms of test parameters, range of normality, and criteria for abnormality (1-14).

\section{ABNORMAL TEST RESULTS AND CLINICAL IMPLICATIONS}

Electrophysiologic signal abnormalities may appear as (a) waves with abnormal amplitude, latency, duration, or morphology; (b) waves recurring with abnormal repetition rate or pattern; (c) waves that emerge de novo due to pathological processes, or (d) wave patterns that are incompatible with the mode of activation or the patient's physiologic state or age (Table 1.5). Each of these macroscopic signal abnormalities can be traced to a disturbance in the activity of microscopic SGUs or to an alteration in the properties of the volume conductor (43).

Signal amplitude depends on the number of SGUs activated in synchrony and on the fidelity of signal propagation from the generator to the recording electrode. An abnormally low amplitude indicates loss of SGUs (e.g., low SNAP amplitude with nerve fiber loss, EEG attenuation with pyramidal cell loss), a decrease in the number of activated SGUs (e.g., low CMAP amplitude with nerve fiber loss or conduction block), desynchrony in SGU activity (e.g., low CMAP amplitude due to temporal dispersion of MFAPs), or changes in the conducting medium increasing the impedance or distance between the generator and electrode (e.g., edema, blood). An abnormally high amplitude indicates an increase in the number of SGUs activated (e.g., giant MUAP with muscle reinnervation), abnormal hypersynchrony of SGUs (e.g., epileptiform EEG/MEG waves), or the presence of a low-impedance path of signal propagation from the generator to the recording electrode (e.g., breach rhythm due to skull defect) (43).

Signal conduction time, the time between initial and subsequent signal generation via nonsynaptic and synaptic mechanisms, is quantified as absolute latency or interpeak latency in the tracing (43). An abnormally long latency indicates conduction slowing. For example, an increase in SNAP, CMAP, or SSEP wave latency is seen with peripheral nerve demyelination; an increase in VEP P100 latency with optic nerve demyelination; and an increase in BAEP III-V and SSEP N13-N20 interpeak latencies with central tract demyelination. Mild prolongation in latency may occur with temporal dispersion and asynchronous activation of SGUs. Since an abnormally short latency cannot easily be attributed to a pathologic process, a technical explanation should be sought to explain this finding (43).

Wave morphology or configuration is influenced by the duration or frequency, the number of phases or turns, and the contour of waveform components. A normal CNP waveform has a characteristic configuration. For example, SNAPs and VEP P100 waves are typically triphasic, whereas CMAPs and EEG vertex waves are usually biphasic. Because most waveforms have a wide range of acceptable configurations, spatial distribution and other wave characteristics must always be considered in deciding if a wave is normal. An increase in wave duration and number of phases imply asynchronous SGU activation (e.g., temporal dispersion from demyelination). Conversely, shorter wave duration and apiculate contour indicate SGU hypersynchrony (e.g., EEG/MEG epileptiform sharp waves).

Wave activation, modulation, and repetition pattern reflect recurrent SGU activity resulting from the intrinsic oscillatory behavior of SGUs and the extrinsic influences of other circuit components (44). The pattern of abnormal activation and recruitment may be simple (e.g., MUAP recruitment is reduced with motor neuron or nerve pathology and increased with muscle pathology) 
or complex (e.g., loss of pyramidal cell afferent input suppresses some and releases other activation patterns, such as slow wave rhythms). So-called abnormal spontaneous activity indicates hyperexcitability of SGUs (e.g., fibrillation potentials due to muscle denervation hypersensitivity), of non-SGU circuit components (e.g., fasciculation potentials from nerve irritability), or of the entire functional circuit including SGUs (e.g., epileptiform activity). Perturbations in network dynamics may appear as rhythmic/periodic waves with abnormal repetition rate (e.g., slower rhythms are seen in the EEG/MEG tracings with encephalopathy).

Spatial distribution is demonstrated by recording the signal from multiple body locations. Pathologic processes that disturb the time-domain characteristics of a signal can also affect the signal's spatial characteristics. It is always prudent to exclude technical factors (e.g., improper electrode placement) and normal physiologic changes (e.g., anterior displacement of the EEG alpha rhythm in drowsiness) before attributing spatial abnormalities to pathological processes. Lastly, signals with time-domain and spatial characteristics that appear "normal" are considered abnormal if their characteristics do not conform to the age and physiologic state of the patient (Table 1.5).

Modality-specific CNP tests are performed to assess the functional integrity of signaling pathways. Most of these tests can also assess specific parts of the pathway to determine which structures are involved in the pathologic process. A general scheme for classifying electrophysiologic test abnormalities was described earlier (Table 1.5). We will now describe the salient abnormalities encountered in each test category and their clinical implications (Table 1.6).

EEG and MEG are sensitive to pathologic processes that implicate the cerebral cortex, either directly or indirectly through its connections with the diencephalon/brainstem. By and large, abnormal EEG and MEG waveforms have the same clinical implications. However, differences in the physics of electrical and magnetic fields prevent us from extrapolating all EEG "interpretation rules" to MEG. Abnormal EEG/MEG waves are either epileptiform or nonepileptiform. Epileptiform activity indicates ongoing epileptic disturbances of the brain, either interictal (spikes or sharp waves) or ictal (seizures) (45). Nonepileptiform abnormalities include changes in background rhythm, abnormal slow waves, abnormal response to activation, and asymmetries in amplitude or other parameters (46). As a rule, focal nonepileptiform abnormalities indicate localized cerebral dysfunction if intermittent and structural lesions if persistent while diffuse bihemispheric changes imply encephalopathy, encephalitis, or neurodegeneration. Periodic discharges are either epileptiform or nonepileptiform (47) and, if epileptiform, they are either ictal, interictal, or due to an epileptic disturbance that lie somewhere along the ictal-interictal continuum (48).

EMG and NCS have been, and are still, the best tools for assessing peripheral nerve and muscle physiology. Needle EMG findings, particularly abnormalities in MUAP morphology and recruitment pattern, can distinguish a myopathy from a neuropathy or motor neuronopathy. Abnormal spontaneous activity implies nerve fiber irritability if motor units are involved and denervation hypersensitivity if individual muscle fibers are affected. Increased jitter in single-fiber EMG is diagnostic of a neuromuscular junction disorder. The pattern of CMAP/SNAP amplitude and the latency abnormalities in NCS will clarify if a neuropathy is focal, multifocal, or diffuse; if it is sensory, motor, or mixed; and if it is primarily due to demyelination or axonal loss. CMAP amplitude increment/decrement with repetitive nerve stimulation can differentiate a presynaptic from a postsynaptic neuromuscular junction disorder. If the routine NCS is normal, the finding of abnormal $F$ waves implies more proximal involvement of the motor pathway (e.g., a radiculopathy or motor neuronopathy). Abnormal $H$, blink, or long-latency reflexes indicate peripheral nerve pathology or a lesion in the intra-axial segment of the reflex pathway.

$E P$ and ERP can detect a disease in its early stages if it involves structures in the signaling pathway. Conduction slowing increases absolute latency if present between the sensory receptor and EP generator (e.g., peripheral nerve demyelination) and increases interpeak latency if present between two EP generators (e.g., central tract demyelination). A loss of amplitude is particularly significant if it represents a left-right asymmetry or a relative change from baseline, such as during intraoperative monitoring (IOM). Giant SSEP or VEP cortical potentials indicate cortical hyperexcitability (46). Impairment in attentional or cognitive processing from neurodegeneration or psychopathology may increase P300 latency or decrease P300 amplitude, or it may result in abnormal MN morphology (47). Brain disorders that cause psychomotor retardation often manifest as an early decrease in BSP or CNV amplitude.

Autonomic function tests evaluate cardiovagal, adrenergic, and sudomotor autonomic functions. In $H R-D B$, disorders that impair the cardiovagal reflex manifest as reduced or absent HR with deep breathing. Certain abnormal BP and HR patterns during HUT or VAL are valuable for diagnosing sympathetic dysfunction or generalized dysautonomias due to diabetes mellitus, amyloidosis, and other diseases $(6,34)$. In QSART, low sweat output indicates sympathetic sudomotor failure, as in distal small-fiber neuropathy and idiopathic anhidrosis, and high sweat output indicates sweat gland hypersensitivity, as in reflex sympathetic dystrophy. The pattern of anhidrosis and hyperhidrosis in TST allows the differential diagnosis to be narrowed down to a few dysautonomias $(6,34)$.

Sleep studies involve multimodal tracking of sleep stage to detect abnormalities in sleep latency, efficiency, and architecture (7). In PSG, other physiologic disturbances can also be detected, such as apneas, increased airway resistance, blood oxygen desaturation, cardiac arrhythmias, abnormal movements, and parasomnias. During MNM, changes in ICP, cerebral blood flow, brain tissue oxygenation, and/or biochemical markers of brain metabolism indicate impending brain injury or exacerbation of previous injury $(9,35)$. 
TABLE 1.6: Abnormal Neurophysiologic Test Results and Their Clinical Implications

\section{EEG/MEG}

Epileptiform activity-epileptic brain disturbance with or without structural brain lesion or encephalopathy

Periodic discharges-epileptic brain disturbance often with structural brain lesion or encephalopathy

Altered background rhythm-usually encephalopathy if generalized and structural brain lesion if focal

Abnormal slow-wave activity-usually encephalopathy if generalized and structural brain lesion if focal

Abnormal response to activation-usually encephalopathy if generalized and structural brain lesion if focal

Amplitude and other asymmetries-structural brain lesion if persistent and physiologic disturbance if transient

\section{Needle EMG}

Abnormal insertional activity-increased with muscle fiber irritability and decreased with muscle fiber loss

Abnormal spontaneous activity-muscle fiber denervation hypersensitivity or motor neuron or nerve irritability

High-amplitude, short-duration MUAPs-neuropathic MUAPs with collateral reinnervation of muscle fibers

Low-amplitude, long-duration MUAPs-myopathic MUAPs with loss of muscle fibers

Decreased recruitment of MUAPs-motor neuron or nerve fiber pathology preventing motor unit activation Increased recruitment of MUAPs-loss of muscle fibers with compensatory early motor unit recruitment Increased jitter with single-fiber EMG-neuromuscular junction disorder

\section{NCS}

Decreased CMAP amplitude-motor neuron or axonal loss, motor conduction block or temporal dispersion

Decreased SNAP amplitude-sensory axonal loss, sensory conduction block or temporal dispersion

Increased CMAP latency-motor conduction slowing with demyelination of large motor nerve fibers

Increased SNAP latency-sensory conduction slowing with demyelination of large sensory nerve fibers

Increased $\mathrm{F}$ wave latency-motor conduction slowing with demyelination of distal or proximal motor nerve fibers

Absent $\mathrm{F}$ waves-conduction failure due to motor nerve or axon pathology or to motor conduction block

Absent $\mathrm{H}$ reflex-conduction failure due to a lesion in the sensory, central, or motor part of the stretch reflex circuit

Abnormal repetitive stimulation-presynaptic or postsynaptic disorder of neuromuscular junction transmission

EP

Increased absolute latency-delayed signal conduction from peripheral stimulation site to EP generator

Increased interpeak latency-delayed signal conduction between two EP generators due to a central tract disorder

Decreased EP amplitude-conduction failure in central tract fibers (e.g., compression or ischemia) or loss of SGUs

Increased EP amplitude-hypersynchrony (e.g., giant SSEPs or VEPs with cortical hyperexcitability)

Absent EP response-generator dysfunction or disconnection from signaling pathway

Abnormal wave configuration-most of the above processes will also affect wave morphology and duration 
TABLE 1.6: Abnormal Neurophysiologic Test Results and Their Clinical Implications (continued)

ERP

Increased P300 latency-cognitive dysfunction due to dementia or psychiatric disorder

Decreased P300 amplitude-cognitive dysfunction due to dementia or psychiatric disorder

Altered MN morphology-attentional perceptual processing deficit due to dementia or psychiatric disorder

Decreased amplitude of BSP early components-psychomotor retardation due to psychiatric or movement disorder

Decreased amplitude of CNV late component-psychomotor retardation due to psychiatric or movement disorder

\section{Autonomic testing}

Reduced or absent HR-DB-impairment of cardiovagal reflex

Abnormal HR response to VAL-impairment of vagal component of baroreflex

Abnormal BP response to HUT/VAL-sympathetic adrenergic dysfunction (e.g., generalized dysautonomias)

Reduced sweat secretion rate in QSART-sympathetic sudomotor dysfunction (e.g., distal small-fiber neuropathy)

Increased sweat secretion rate in QSART-sympathetic sudomotor hyperactivity (e.g., reflex sympathetic dystrophy)

Abnormal sweat patterns in TST-anhidrosis or hyperhidrosis (e.g., generalized dysautonomias)

\section{Sleep studies}

Abnormal sleep continuity or pattern-impairment in chronophasic, homeostatic, and/or afferent sleep-wake drives

Decreased or absent respiratory airflow and/or effort-increased upper airway resistance, obstructive/central apnea

Blood oxygenation desaturation-hypoxia from obstructive/central apnea, cardiac insufficiency, etc.

ECG changes-tachyarrhythmia or bradyarrhythmia from sleep apnea, cardiac disease, etc.

Anterior tibialis EMG-periodic limb disorder of sleep, other movement disorders

\section{Neuromonitoring}

Elevation of ICP-cerebral edema, space-occupying lesion, impaired autoregulation, abnormal CSF flow dynamics

Abnormal cerebral blood flow-increased ICP, vasospasm, impaired autoregulation, reperfusion

Abnormal brain oxygenation-ischemia/hyperemia, abnormal oxygen extraction, flow-metabolism decoupling

Abnormal biochemistry-impaired energy-substrate metabolism, cell injury, cell membrane breakdown

Note: Listed for each test modality are the most commonly encountered test result abnormalities and associated pathological processes, not specific diseases.

BSP, Bereitschaftspotential; CMAP, compound motor action potential; CNV, contingent negative variation; CSF, cerebral spinal fluid; EMG, electromyography; EP, evoked potential;

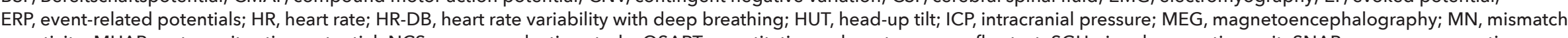
negativity; MUAP, motor unit action potential; NCS, nerve conduction study; QSART, quantitative sudomotor axon reflex test; SGU, signal-generating unit; SNAP, sensory nerve action potential; SSEP, somatosensory evoked potentials; TST, thermoregulatory sweat test; VAL, Valsalva; VEP, visual evoked potentials. 


\section{ARTIFACT RECOGNITION AND OTHER TECHNICAL CHALLENGES}

Although the terms "artifact" and "noise" are used interchangeably, an artifact is, strictly speaking, the graphical depiction of noise entering the system and mixing with the desired signal (Table 1.7). Simply put, noise is the undesired analog signal and artifact is the undesired digital trace or waves. Separating noise from desired signal is achieved with modern technology and proper technique $(25,26,49,50)$. The use of finely engineered signal transducers and recording equipment, the optimal placement of electrodes and sensors, proper grounding, and the ability to recognize and avoid potential sources of noise are crucial for maximizing the signal-to-noise ratio during neurophysiologic testing (Figure 1.1).

Despite the best technology and technique, some amount of noise will still enter the recording system and appear in the record as an artifact. Separating artifact from "useful waves" is accomplished by direct inspection of the record and, if necessary, by additional digital signal processing (26). Visual analysis involves artifact recognition based on the spatiotemporal characteristics of waves. Depending on the test, postacquisition digital signal processing, such as averaging, artifact rejection, digital filtering, and quantitative analysis, may be performed to eliminate or reduce artifact (see Chapter 2).

Artifact classification is based on the physical properties and source of noise causing the artifact and on the mechanism by which noise gains access to the recording system (Table 1.7) $(25,27,49)$. Any part of the recording system is a potential portal for noise but the most vulnerable sites are the movable wires outside the chassis of the recording apparatus and the interface between machine and body where transduction of biological signals to electronic current takes place. The electrode-tissue interface is usually the site of biological to electronic signal transduction during CNP testing (50).

Environmental and instrument noise originates from sources outside the patient's body (49). In the hospital, the most significant source of environmental noise is alternating current (AC) from wires and equipment in the building $(27,49)$. AC noise entering the recording system, via its inductive effects on electrode wires or via poorly applied electrodes, result in $60-\mathrm{Hz}$ artifact
(50 Hz in Europe) (27). Electric-powered equipment in the room or building, such as radiological scanners and surgical devices, may contribute to environmental noise $(27,49)$. Instrument noise, such as thermal noise and amplifier noise, originates from the electronics of the recording apparatus. Good engineering design keeps instrument noise to a minimum but it can be an issue if the equipment is old, malfunctioning, or poorly maintained (49).

Noise generated at the signal transduction site, usually the electrode-tissue interface, is a common source of artifacts in CNP $(49,50)$. Slight movement of electrodes and other instabilities in the electrode-tissue interface, such as electrode polarization or impedance mismatch, may affect charge distribution and currents at the electrode-tissue interface and lead to signal distortion or electrode artifact (50). Gross movements of multiple electrodes, wires, or cable connections may appear as a movement artifact in the record. An electrode with high impedance can be "replaced" by the ground electrode, resulting in ground lead artifact.

Medical devices that deliver current to the patient can be the source of artifacts $(25,49)$. In some neurophysiologic studies (e.g., NCS, EP), the stimulus artifact indicates the time of stimulation, but excessive stimulus artifacts can interfere with and distort the signal $(3,16)$. Implanted medical devices, such as pacemakers and stimulators, produce characteristic artifacts. During CNP testing, some normal physiological processes in the patient's body may be captured in the record as physiological artifacts (27). Note that a waveform can represent desired signal in one test or in one channel and the same waveform can be a physiological artifact in another test or in a different channel in the same record.

Other technical issues encountered in CNP include (a) improper placement of recording electrodes causing artifactual changes in signal or signal distortion; (b) improper stimulus delivery leading to insufficient activation, cross-activation of other structures, or contamination by stimulus artifact: and (c) failure to recognize the cause of signal distortion, such as temperature, humidity, and other physical factors, the presence of a low-impedance path or an intervening substance between the generator and recording electrode, or technical factors, such as poor equipment maintenance and calibration (Table 1.7). This book and atlas will provide students and professionals of CNP a deep understanding of artifacts and technical issues that are encountered during each of the CNP tests introduced in this chapter. 
TABLE 1.7: Sources of Aartifacts in Clinical Neurophysiologic Testing

Environmental sources-electric, magnetic, or electromagnetic field from wires and equipment in the building AC noise-AC artifact: 60-cycle artifact in the United States, 50-cycle artifact in Europe

Scanners and other equipment-AC artifact, high-frequency artifact

Fluorescent light-if light intensity is strong it can contribute to AC artifact

Strobe light-artifact is time-locked to the flash and reflects the photic frequency of the source

Television and video monitors-usually high-frequency artifacts (e.g., $300 \mathrm{~Hz})$

Diathermy or cautery device-usually high-frequency artifacts (e.g., $120 \mathrm{~Hz}$ )

Dripping fluid-inductive effects on electrode wires result in artifact (e.g., intravenous fluid drip artifact)

Radiofrequency noise-audible and can often be identified (e.g., paging system, phone ringing)

Recording instrument-electronic circuitry and components of the recording apparatus

Thermal noise-random motion of charges in any part of the apparatus may result in high-frequency artifact

Shot noise-charges crossing a junction (e.g., $n-p$ junctions in a transistor, may result in artifact)

Equipment wiring noise-AC noise from wires in apparatus; produces $60-\mathrm{Hz}$ artifact ( $50 \mathrm{~Hz}$ in Europe)

Amplifier noise-intrinsic noise of an operational amplifier

Filter noise-ringing artifacts that appear as spurious signals near sharp transitions

Quantization noise-arises from digitization; often negligible with respect to other sources of noise

Switching artifact-opening and closing of control switches on equipment

Signal transduction site-electrochemical change at the interface between the probe and the patient's tissue Increased impedance-loss of signal, electrode artifacts, AC artifact, and other artifacts

Poor electrode contact-electrode can be "replaced" by ground electrode resulting in ground lead artifact Impedance mismatch-reduced cancellation of common-mode noise resulting in AC and other artifacts

Salt bridge or short circuit-increased cancellation resulting in reduced signal amplitude or signal loss Electrode polarization-altered charge distribution and current at the interface resulting in signal distortion Junctional potential-altered charge distribution and current at the interface resulting in signal distortion Slight electrode movement-altered charge distribution resulting in signal distortion or electrode artifact Gross movement-multiple electrodes, wires, or cable box connections affected resulting in movement artifact Mechanical vibrations-motion artifact from ventilator, compressors, and other hospital equipment

Current delivering devices-external or implanted medical device injecting current to the patient's body Neurodiagnostic stimulator-excessive stimulus artifact can interfere with the signal (e.g., SNAP or CMAP) Implanted cardiac device-distinctive artifact frequency and morphology (e.g., pacemaker/defibrillator artifact) Implanted nerve stimulator-distinctive artifact frequency and morphology (e.g., vagus nerve stimulator) Transcutaneous stimulator-distinctive artifact frequency; transcutaneous electrical nerve stimulation 
TABLE 1.7: Sources of Artifacts in Clinical Neurophysiologic Testing (continued)

\section{Normal physiological processes-normal activity of biological structures in the patient's body}

Eye movement-eye blink, slow lateral eye movements, eye flutter, and other optokinetic artifacts

Oropharyngeal activity-glossokinetic, talking, biting, chewing, teeth-grinding, snoring, and other artifacts

Skeletal muscle activity-EMG artifact, tremor artifact, photomyogenic artifact, motor artifact in SNAP

Cardiovascular activity-ECG artifact, pulse artifact, cardioballistic artifact

Sudomotor activity-sweat artifact, galvanic skin response or electrodermal artifact

Movement of body parts-movement artifacts (see Signal transduction site section)

\section{Other technical factors-not considered noise but can result in artifactual signal distortion}

Improperly placed electrodes-artifactual change in signal amplitude, latency, and other characteristics

Suboptimal stimulus delivery-insufficient activation, cross-activation of other structures, stimulus artifact

Altered volume conduction-fluid or substance between generator and electrode, low impedance path

Temperature effects-artifactual change in amplitude and latency if not recognized and remedied

Humidity and other physical factors-e.g., OSART is sensitive to humidity

Poor equipment maintenance-uncalibrated apparatus, old corroded sensors

Noise entering the recording system may appear as an artifact in the record. Potential sources of noise during clinical neurophysiological testing include (a) electrical, magnetic, or

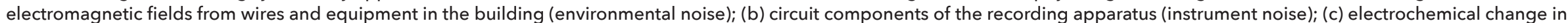

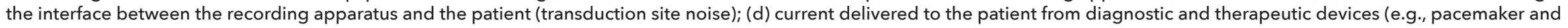

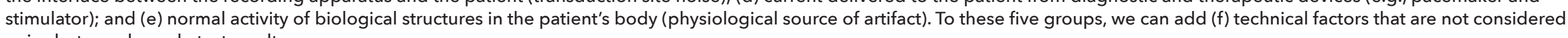
noise but can degrade test results.

$A C$, alternating current; CMAP, compound motor action potential; EMG, electromyography; QSART, quantitative sudomotor axon reflex test; SNAP, sensory nerve action potential.

\section{REFERENCES}

1. Ebersole JS, Husain AM, Nordli DR, eds. Current Practice of Clinical Electroencephalography. 4th ed. Philadelphia, PA: Wolters Kluwer Health; 2014.

2. Papanicolaou AC, ed. Clinical Magnetoencephalography and Magnetic Source Imaging. Cambridge, UK: Cambridge University Press; 2009.

3. Kimura J. Electrodiagnosis in Diseases of Nerve and Muscle: Principles and Practice. 4th ed. New York, NY: Oxford University Press; 2013.

4. Husain AM. Evoked potentials overview. In: Ebersole JS, Husain AM, Nordli DR, eds. Current Practice of Clinical Electroencephalography. 4th ed. Philadelphia, PA: Wolters Kluwer Health; 2014:442-487.
5. Luck SJ. An Introduction to the Event-Related Potential Technique. 2nd ed. Cambridge, MA: MIT Press; 2014.

6. Benarroch EE, ed. Autonomic Neurology. New York, NY: Oxford University Press; 2014.

7. Chokroverty S, Radtke R, Mullington J. Polysomnography: technical and clinical aspects. In: Schomer DL, Lopes da Silva FH, eds. Niedermeyer's Electroencephalography: Basic Principles, Clinical Applications, and Related Fields. 6th ed. Philadelphia, PA: Lippincott Williams \& Wilkins; 2011:817-864.

8. Husain AM, ed. A Practical Approach to Neurophysiologic Intraoperative Monitoring. New York, NY: Demos Medical Publishing; 2008. 
9. Le Roux PD, Levine JM, Kofke WA. Monitoring in Neurocritical Care. Philadelphia, PA: Elsevier; 2013.

10. He B, Liu Z. Multimodal functional neuroimaging: integrating functional MRI and EEG/MEG. IEEE Rev Biomed Eng. 2008;1:23-40. doi:10.1109/ RBME.2008.2008233.

11. American Clinical Neurophysiology Society (ACNS) Guidelines and Consensus Statements. American Clinical Neurophysiology Society website. https:/ /www.acns.org/practice/guidelines. Updated: August 11, 2016.

12. Deuschl, G, Eisen A, eds. Recommendations for the practice of clinical neurophysiology: guidelines of the International Federation of Clinical Neurophysiology. Electroencephalogr Clin Neurophysiol Suppl. 1999;52:1-304.

13. American Association of Neuromuscular \& Electrodiagnostic Medicine (AANEM) Practice Guidelines and Quality. American Association of Neuromuscular \& Electrodiagnostic Medicine (AANEM) website. http:// www.aanem.org/Practice/Guidelines-and-Performance-Measures. .

14. American Academy of Neurology. Assessment: clinical autonomic testing report of the Therapeutics and Technology Assessment Subcommittee of the American Academy of Neurology. Neurology. 1996;46(3):873-880.

15. Malmivuo J. Comparison of the properties of EEG and MEG in detecting the electric activity of the brain. Brain Topogr. 2012;25(1):1-19. doi:10.1007/ s10548-011-0202-1.

16. Preston DC, Shapiro BE, eds. Electromyography and Neuromuscular Disorders: Clinical-Electrophysiological Correlations. 3rd ed. London, UK: Elsevier Saunders; 2013.

17. Hallett M. Electrophysiologic evaluation of movement disorders. In: Aminoff MJ, ed. Aminoff's Electrodiagnosis in Clinical Neurology. 6th ed. Amsterdam, the Netherlands: Elsevier Saunders; 2012:437-454.

18. Emerson RG. Somatosensory evoked potentials. In: Levins KH, Luders HO, eds. Comprehensive Clinical Neurophysiology. Philadelphia, PA: W.B. Saunders Company; 2000:543-564

19. Epstein CM. Visual evoked potentials. In: Levins $\mathrm{KH}$, Luders HO, eds. Comprehensive Clinical Neurophysiology. Philadelphia, PA: W.B. Saunders Company; 2000:507-524.

20. Luders HO, Terada K. Auditory evoked potentials. In: Levins KH, Luders HO, eds. Comprehensive Clinical Neurophysiology. Philadelphia, PA: W.B. Saunders Company; 2000:525-541.

21. Galloway GM, Nuwer MR, Lopez JR, et al., eds. Intraoperative Neurophysiologic Monitoring. Cambridge, UK: Cambridge University Press; 2010.

22. Nuwer MR, Emerson RG, Galloway G, et al. Evidence-based guideline update: intraoperative spinal monitoring with somatosensory and transcranial electrical motor evoked potentials. J Clin Neurophysiol. 2012;29(1):101-108.doi:10.1212/WNL.0b013e318247fa0e.

23. Lopez da Silva FH. Event-related potentials: general aspects of methodology and quantification. In: Schomer DL, Lopes da Silva FH, eds. Niedermeyer's Electroencephalography: Basic Principles, Clinical Applications, and Related Fields. 6th ed. Philadelphia, PA: Lippincott Williams \& Wilkins; 2011:923-934.

24. Buijs RM, Swaab DF, eds. Autonomic Nervous System. Amsterdam, the Netherlands: Elsevier BV; 2013. Handbook of Clinical Neurology; vol 117 (3rd series).

25. Mainardi LT, Bianchi AM, Cerutti S. Digital biomedical signal acquisition and processing. In: Liang $\mathrm{H}$, Bronzino JD, Peterson DR, eds. Biosignal Processing Principles and Practices. Boca Raton, FL: CRC Press; 2013:1.1-1.23.

26. Lagerlund TD. Digital signal processing. In: Daube JR, Rubin DI, eds. Clinical Neurophysiology. 3rd ed. New York, NY: Oxford University Press; 2009:53-68.

27. Daube JR. Waveforms and artifacts, In: Daube JR, Rubin DI, eds. Clinical Neurophysiology. 3rd ed. New York, NY: Oxford University Press; 2009:103-114.

28. Fisch BJ. Fisch and Spehlmann's EEG Primer: Basic Principles of Digital and Analog EEG. 3rd ed. Amsterdam, the Netherlands: Elsevier; 1999.

29. Chiappa KH. Principles of evoked potentials. In: Chiappa KH, ed. Evoked Potentials in Clinical Medicine. 3rd ed. Philadelphia, PA: Lippincott-Raven; 1997:1-30

30. Tatum WO. Handbook of EEG Interpretation. 2nd ed. New York, NY: Demos Medical Publishing; 2014.

31. Bagić AI, Bowyer SM, Kirsch HE, et al. American Clinical MEG Society (ACMEGS) position statement \#2: the value of magnetoencephalography (MEG)/magnetic source imaging (MSI) in noninvasive presurgical mapping of eloquent cortices of patients preparing for surgical interventions. J Clin Neurophysiol. 2017;34(3):189-195. doi:10.1097/WNP.0000000000000366.

32. Rubin DI. Needle electromyography: basic concepts and patterns of abnormalities. Neurol Clin. 2012;30(2):429-456. doi:10.1016/j.ncl.2011.12.009.

33. Yamada T, Meng E, Seaba P. Principles of evoked potentials. In: Yamada T, Meng E, eds. Practical Guide for Clinical Neurophysiologic Testing: EP, LTM, IOM, PSG and NCS. Philadelphia, PA: Lippincott Williams \& Wilkins; 2011.

34. Low PA, Tomalia VA, Park K. Autonomic function tests: some clinical applications. J Clin Neurol. 2013;9(1):1-8. doi:10.3988/jcn.2013.9.1.1.

35. Kirkman MA, Smith M. Multimodal intracranial monitoring: implications for clinical practice. Anesthesiol Clin. 2012;30(2):269-287. doi:10.1016/ j.anclin.2012.05.007. 
36. Lagerlund TD. Electrophysiological generators in clinical neurophysiology. In: Daube JR, Rubin DI, eds. Clinical Neurophysiology. 3rd ed. New York, NY: Oxford University Press; 2009:97-101.

37. Daube JR, Stead SM. Basic neurophysiology. In: Daube JR, Rubin DI, eds. Clinical Neurophysiology. 3rd ed. New York, NY: Oxford University Press; 2009:69-95.

38. Buzsáki G, Anastassiou CA, Koch C. The origin of extracellular fields and currents-EEG, ECoG, LFP and spikes. Nat Rev Neurosci. 2012;13(6):407-420.

39. Hansman RJ Jr. Characteristics of instrumentation. In: Webster JG, Eren H, eds. Measurement, Instrumentation, and Sensors Handbook: Spatial, Mechanical, Thermal, and Radiation Measurement. 2nd ed. Boca Raton, FL: CRC Press/ Taylor \& Francis Group; 2014:2:1-9.

40. Lagerlund TD, Rubin DI, Daube JR. Volume conduction. In: Daube JR, Rubin DI, eds. Clinical Neurophysiology. 3rd ed. New York, NY: Oxford University Press; 2009:33-51.

41. Rutkove SB. Introduction to volume conduction. In: Blum AS, Rutkove SB, eds. The Clinical Neurophysiology Primer. Totowa, NJ: Humana Press Inc.; 2007:43-53.

42. Stegeman DF, Dumitru D, King JC, et al. Near- and far-fields: source characteristics and the conducting medium in neurophysiology. J Clin Neurophysiol. 1997;14(5):429-442. doi:10.1097/00004691.
43. Quian Quiroga R, Panzeri S. Principles of Neural Coding. Boca Raton, FL: CRC Press; 2013.

44. Ashwin P, Coombes S, Nicks RJ. Mathematical frameworks for oscillatory network dynamics in neuroscience. J Math Neurosci. 2016;6(1):2. doi:10.1186/ s13408-015-0033-6.

45. Mader EC Jr., Olejniczak PW. Epilepsy syndromes. In: Fisch BJ, ed. Epilepsy and Intensive Care Monitoring: Principles and Practice. New York, NY: Demos Medical Publishing; 2010:119-150.

46. Benbadis SR. Abnormal nonepileptiform EEG. In: Tatum WO, ed. Handbook of EEG Interpretation. 2nd ed. New York, NY: Demos Medical Publishing; 2014:57-78.

47. Hirsch LJ, LaRoche SM, Gaspard N, et al. American clinical neurophysiology society's standardized critical care EEG terminology: 2012 version. J Clin Neurophysiol. 2013;30(1):1-27. doi:10.1097/WNP.0b013e3182784729.

48. Rodríguez V, Rodden MF, LaRoche SM. Ictal-interictal continuum: a proposed treatment algorithm. Clin Neurophysiol. 2016;127(4):2056-2064. doi:10.1016/j.clinph.2016.02.003.

49. Semmlow J. Signals and systems: Noise and variability. In: Ratner BD, Hoffman AS, Schoen FJ, et al., eds. Biomedical Engineering Desk Reference. Oxford, UK: Academic Press; 2009:73-76.

50. Button V. Principles of Measurement and Transduction of Biomedical Variables. Oxford, UK: Elsevier; 2015. 